\title{
Scalar excursions in large-eddy simulations
}

\author{
Georgios Matheou
}

Jet Propulsion Laboratory, California Institute of Technology, Pasadena, California 91109, USA

\author{
Paul E. Dimotakis
}

Graduate Aerospace Laboratories, California Institute of Technology, Pasadena, California 91125, $U S A$

\begin{abstract}
The range of values of scalar fields in turbulent flows is bounded by their boundary values, for passive scalars, and by a combination of boundary values, reaction rates, phase changes, etc., for active scalars. The current investigation focuses on the local conservation of passive scalar concentration fields and the ability of the large-eddy simulation (LES) method to observe the boundedness of passive scalar concentrations. In practice, as a result of numerical artifacts, this fundamental constraint is often violated with scalars exhibiting unphysical excursions. The present study characterizes passive-scalar excursions in LES of a shear flow and examines methods for diagnosis and mitigation of the problem. The analysis of scalar-excursion statistics provides support of the main hypothesis of the current study that unphysical scalar excursions in LES result from dispersive errors of the convection-term discretization where the subgrid-scale model (SGS) provides insufficient dissipation to produce a sufficiently smooth scalar field. In the LES runs three parameters are varied: the discretization of the convection terms, the SGS model, and grid resolution. Unphysical scalar excursions decrease as the order of accuracy of non-dissipative schemes is increased, but the improvement rate decreases with increasing order of accuracy. Two SGS models are examined, the stretched-vortex and a constant-coefficient Smagorinsky. Scalar excursions strongly depend on the SGS model. The excursions are significantly reduced when the characteristic SGS scale is set to double the grid spacing in runs with
\end{abstract}


the stretched-vortex model. The maximum excursion and volume fraction of excursions outside boundary values show opposite trends with respect to resolution. The maximum unphysical excursions increase as resolution increases, whereas the volume fraction decreases. The reason for the increase in the maximum excursion is statistical and traceable to the number of grid points (sample size) which increases with resolution. In contrast, the volume fraction of unphysical excursions decreases with resolution because the SGS models explored perform better at higher grid resolution. 


\section{Introduction}

The range of values of scalar fields in turbulent flows is typically bounded by their boundary values, for passive scalars, and the combination of boundary values, reaction rates, phase changes, etc., for active scalars. The transport and conservation of mass-fraction fields $Y_{\alpha}(\mathbf{x}, t)$ are governed by the general species-transport equation for each of the $\alpha=1, \ldots, n$ species (e.g., Dimotakis, 2005),

$$
\frac{\partial \rho Y_{\alpha}}{\partial t}+\frac{\partial\left(u_{j}+v_{\alpha j}\right) \rho Y_{\alpha}}{\partial x_{j}}=\omega_{\alpha}
$$

where $u_{i}(\mathbf{x}, t)$ is the local (mass-averaged) flow velocity field, $v_{\alpha i}$ is the $\alpha$-species diffusion velocity in the local $u$-frame, and $\omega_{\alpha}$ is the local $\alpha$-species net production rate (chemical, nuclear, biological, phase-change, etc.). If species-concentration gradients contribute the dominant diffusive-flux component, we may write, to lowest order,

$$
v_{\alpha i}=-\mathcal{D}_{\alpha} \frac{\partial \ln Y_{\alpha}}{\partial x_{i}}
$$

where $\mathcal{D}_{\alpha}$ is the local multicomponent $\alpha$-species diffusivity. If we also assume, for the purposes of illustration, that fluid density can be treated as uniform and that there are no local species production/consumption rates, the species-transport equations simplify to the familiar (Fickian) species-conservation equations, i.e.,

$$
\left(\frac{\partial}{\partial t}+u_{j} \frac{\partial}{\partial x_{j}}\right) Y_{\alpha}=\frac{\partial}{\partial x_{i}}\left(\mathcal{D}_{\alpha} \frac{\partial Y_{\alpha}}{\partial x_{i}}\right)
$$

In a situation where we may approximate scalar transport in terms of a mixture-fraction field, $Z(\mathbf{x}, t)$, such as in shear-layer mixing of simple fluids in uniform-density flow, in which $Z$ represents the fraction of, say, high-speed fluid, and only binary diffusion is active with a uniform diffusion coefficient, $\mathcal{D},(3)$ simplifies further to,

$$
\left(\frac{\partial}{\partial t}+u_{j} \frac{\partial}{\partial x_{j}}\right) Z=\mathcal{D} \frac{\partial}{\partial x_{i}} \frac{\partial Z}{\partial x_{i}}
$$


or,

$$
\frac{\mathrm{D} Z}{\mathrm{D} t}=\mathcal{D} \frac{\partial^{2} Z}{\partial x_{i}^{2}}
$$

Equation (5), which we will use to illustrate the effects in flow environments where it is valid, has a convective (Lagrangian) operator on the left-hand side. Absent diffusion, i.e., if $\mathcal{D}=0$, the left-hand side simply transports to the flow interior mixture-fraction (scalar) values provided by the boundary/inflow conditions. In the shear-layer example, the resulting scalar field would intersperse values of $Z=1$, corresponding to fluid originating in the high-speed stream, and $Z=0$, corresponding to fluid originating from the low-speed stream, and no intermediate values would be encountered.

For $\mathcal{D}>0$, the right-hand side in (5) is a local averaging operator and can only produce values at $(\mathbf{x}, t)$ that are intermediate to bounding values in its periphery. As a consequence, in the presence of diffusion, however small, the mixture-fraction scalar field is bounded throughout the domain and, in our shear-layer example, limited to a span of values,

$$
0 \leq Z(\mathbf{x}, t) \leq 1
$$

There are many consequences of this boundedness constraint (e.g., Dimotakis and Miller, 1990) whose discussion is beyond the scope of the present paper. The scalar boundedness property (6) is independent of the velocity field and holds for any velocity field $u_{j}$ in (4). Moreover, the boundedness property holds in any $n_{d}$-dimensional space, where $n_{d}$ is the number of spatial dimensions.

More complicated situations can arise in which local-acceleration effects on mixtures of fluids with different molar masses can induce segregation (unmixing) that can give rise to legitimate excursions outside the bounds of (6). Multi-species and differential-diffusion effects can also introduce additional complications (Dimotakis, 2005). Such effects are ignored in the present study for the purposes of illustrating the fundamental issues that arise even in the simplest cases.

Returning to simple flows, numerical simulation should respect the fundamental conservation laws, i.e., mass and momentum conservation for incompressible flows, and physical attributes of the flow fields, such as the scalar boundedness property. When the continuous versions of the equations 
of motion are discretized on a numerical grid, careful considerations must be made for the discrete system to preserve the conservation and other properties of the continuous operators (see $\S 2.2$ ).

The deviation of the discrete solution from the exact solution of the continuous system can be described by the general term of "model error." Model error can be divided in two broad categories: (i) discretization errors that can include truncation, dispersion, aliasing, and artificial numericaldissipation errors, and (ii) physical-model errors, such as those arising from inaccurate modeling of the fluid dynamics, the transport of various quantities, and turbulence by a subgrid-scale closure.

The importance and contribution of these two model error types varies in different approaches. For instance, in direct numerical simulation (DNS), the discretization error dominates, whereas in Reynolds-averaged Navier-Stokes (RANS) formulations model-error is mostly attributable to turbulence parameterization schemes. In large-eddy simulation (LES), because a wide range of motions is resolved and a strong interaction between resolved and SGS dynamics takes place, there is a significant interplay between the two error types. Previous studies (Ghosal, 1996; Vreman et al., 1996; Kravchenko and Moin, 1997; Fedioun et al., 2001; Chow and Moin, 2003; Geurts, 2009) investigated model error in LES pertaining to predictions of the velocity field.

The present study addresses model error in LES of scalar transport and mixing. Specifically, we focus on the local conservation of scalar concentration fields and the ability of LES to observe the boundedness of the scalar concentration, as diagnosed by unphysical excursions outside the limits on the (local and global) boundary surfaces. In practice, this fundamental constraint is often violated with scalar excursions exhibiting under- and over-shoots in their values, i.e., $Z<0$ and $Z>1$, respectively, that are unphysical, even though for a well-designed method, overall scalars, such as mole or mass fractions, may be globally conserved. This common numerical artifact may not be diagnosed and is not often reported, and only few characterizations are available (Cook et al., 2004; Matheou et al., 2010; Mattner, 2011).

The goal of the current investigation is to characterize passive-scalar excursions in LES and examine methods for diagnosis and mitigation of the problem. The scalar conservation and boundedness in LES is discussed in $\S 2.1$ and a working hypothesis is presented in $\S 2.2$. To help characterize scalar excursions a specific shear flow is considered and the investigation includes the three main 
parameters in LES: the subgrid-scale (SGS) model, convection discretization, and grid resolution. Two SGS models are used: the constant-coefficient Smagorinsky (1963) and the stretched-vortex model (Misra and Pullin, 1997; Pullin, 2000; Voelkl et al., 2000). Several convection schemes with diverse properties are used, including a spectral method, (non-dissipative) fully conservative schemes of various orders, a linear (dissipative) upwinding scheme, and a (dissipative) flux-limited monotone discretization. Two types of diagnostics for scalar excursions are defined in $\S 3$. Scalar excursion statistics are presented in $\S 4$, followed by discussion and conclusions.

\section{Large-eddy simulation}

\subsection{Governing equations}

In most turbulent flows encountered in nature and in engineering applications, the mixture fraction in (4) exhibits a large range of spatial and temporal fluctuations. The same holds for the components of the velocity field, thus a direct integration of the governing equations is impractical for current computing capabilities. Large-eddy simulation is a modeling methodology that tackles this problem by limiting the range of spatial (and temporal) scales that are explicitly computed. This is formally achieved by applying a spatial filter. $\bar{\phi}(\mathbf{x}, t)$ denotes the filtered variable. Presently, the computational domain is triply periodic (without any solid boundaries) and the grid spacing is uniform and isotropic, thus anisotropic filter effects or commutation errors are excluded.

The LES equations for mass, momentum and a passive scalar for an incompressible (constant density) fluid are:

$$
\begin{gathered}
\frac{\partial \bar{u}_{i}}{\partial x_{i}}=0 \\
\frac{\partial \bar{u}_{i}}{\partial t}+\frac{\partial \bar{u}_{j} \bar{u}_{i}}{\partial x_{j}}=-\frac{\partial p^{\prime}}{\partial x_{i}}+\nu \frac{\partial^{2} \bar{u}_{i}}{\partial x_{i}^{2}}-\frac{\partial \tau_{i j}}{\partial x_{j}} \\
\frac{\partial \bar{Z}}{\partial t}+\frac{\partial \bar{u}_{j} \bar{Z}}{\partial x_{j}}=\frac{\nu}{\operatorname{Sc}} \frac{\partial^{2} \bar{Z}}{\partial x_{i}^{2}}-\frac{\partial \sigma_{i}}{\partial x_{i}} .
\end{gathered}
$$

We have replaced the scalar diffusivity $\mathcal{D}$ with the ratio of momentum diffusivity $\nu$ and the Schmidt 
number $\mathrm{Sc} \equiv \nu / \mathcal{D}$. Because of the non-linearity of the equations of motion, the filtering operation results in two additional terms, the SGS tensor $\tau_{i j}=\overline{u_{i} u_{j}}-\bar{u}_{i} \bar{u}_{j} \approx \overline{u_{i}^{\prime} u_{j}^{\prime}}$ in the momentum equation and the SGS flux $\sigma_{j}=\overline{u_{i} Z}-\bar{u}_{i} \bar{Z} \approx \overline{u_{i}^{\prime} Z^{\prime}}$ in the scalar-transport equation. These terms represent the effects of the unresolved, i.e., subgrid scale, dynamics on the evolution of the resolved $\left(\bar{u}_{i}, \bar{Z}\right)$ variables. The functional forms and properties of the SGS terms are discussed in $\S 2.4$.

There are a few important observations regarding the LES system (7). In the present discussion, the filtering procedure is purely formal, i.e., it is used to derive the governing equations, and in the actual LES run, the turbulence model, $\tau_{i j}$ and $\sigma_{i}$, in (7) ensures that all flow scales remain sufficiently resolved on the computational grid. Accordingly, the role of the SGS terms is purely dissipative in the mean. Although some SGS models allow for backscatter, in the present study we only consider forward scatter (dissipation) for simplicity. The presence of backscatter does not change the arguments and conclusions of the present study. The boundedness of the scalar transport equation must also be preserved in the filtered scalar evolution equation, i.e.,

$$
0 \leq \bar{Z} \leq 1
$$

Finally, we note that the LES flow field always exhibits significant fluctuations near the grid scale. In other words, it is "rough," in contrast with a smooth flow field that is the result of a DNS. This is because LES fields are always unresolved in the DNS sense and made "artificially" smooth by the (implicit) LES filter.

\subsection{Convection scheme characteristics, dispersive oscillations, and the Gibbs phenomenon}

In LES, the interaction between resolved and subgrid-scale dynamics is significant and it is expected that both resolved transport and the SGS model will impact scalar-excursion characteristics. Several studies indicate the importance of the convection term discretization on turbulence characteristics in LES (e.g., Horiuti, 1987; Mittal and Moin, 1997; Morinishi et al., 1998; Hill and Pullin, 2004). Presently, we consider another quality metric: passive-scalar boundedness.

Several numerical (discrete) approximations of the continuous momentum and scalar convection 
terms, with diverse properties, are used in the current investigation. The schemes differ in their (artificial) numerical dissipation and can be split into dissipative and non-dissipative, and in their resolving power. The resolving power is the range of wavenumbers that is accurately resolved by a given derivative approximation. Five finite-difference schemes and a pseudo-spectral method are examined and compared.

The non-dissipative schemes are the family of fully conservative approximations of Morinishi et al. (1998). The second-, fourth- and sixth-order schemes are currently used. Their dispersion relations and modified phase speeds are plotted in fig. 1 where the increase in resolving power can be seen as the order of approximation increases. A measure of the resolving power is the number of grid points per wavelength (PPW) required for an error of $\leq 1 \%$ in phase speed (e.g., Lomax et al., 2003). The second-order scheme requires $26 \mathrm{PPW}$, the fourth-order $8 \mathrm{PPW}$, and the sixth-order 5.5 PPW. The increase in the accurately approximated bandwidth becomes progressively smaller as the order of accuracy increases.

All finite-difference discretizations used in the current study are conservative, i.e., the global amount of momentum and scalar can only change because of the boundary conditions. In addition to conservation of the global mean, the Morinishi et al. (1998) schemes conserve kinetic energy and scalar variance, which was shown to be beneficial for turbulent flow simulations. More details and verification of the conservation properties are documented in Appendix A. While the pseudospectral method is not formally conservative, the lack of conservation is compensated by its spectral accuracy.

The main characteristic of the finite-difference schemes of interest in this study is that discrete solutions are dispersive, even for non-dispersive partial differential equations, such as the onedimensional convection of substance $\phi$ with unit velocity, that is instructive to consider, i.e.,

$$
\frac{\partial \phi}{\partial t}+\frac{\partial \phi}{\partial x}=0
$$

For instance, fig. 2 shows the numerical solution of (9) with a rectangular pulse initial condition. The initial condition is regularized by applying an 11-point discrete Gaussian filter, resulting in a maximum product of curvature times $\Delta x$ of 0.57. Accordingly, the initial condition and solution 
are continuous. The domain is periodic in $[0,1]$. Three convection schemes are used, the secondand fourth-order schemes of Morinishi et al. (1998) and an additional scheme with a modified dispersion relation. For the modified scheme, the interpolation weights of Morinish et al. (1998, eq. 101), are replaced by $\beta_{1}=1.4$ and $\beta_{2}=1-\beta_{1}$, instead of $\beta_{1}=9 / 8$. This results in a convection scheme that is second-order accurate with a seven-point stencil and a modified phase speed that is larger than the exact one for small wavenumbers (see fig. 1). This modified second-order scheme qualitatively resembles the tuned centered difference schemes for compressible flow of Hill and Pullin (2004). However, the present modified scheme is used only to illustrate aspects of dispersion errors discussed below and should not be used in a LES.

Because the LES numerical solution is not well-resolved, all schemes exhibit dispersive oscillations near the gradients of $\phi$. Dispersive oscillations develop on opposite sides of the gradients for the standard and modified schemes because of the change in the sign of the phase error. The same behavior is observed for the non-linear LES equations, as discussed in $\S 5$.

The spurious oscillatory behavior of the numerical solution of (9) in fig. 2 is sometimes misinterpreted as a Gibbs oscillation (e.g., Sengupta et al., 2004). The Gibbs phenomenon concerns local-value function construction based on its Fourier expansion. In contrast, in finite-difference dispersive oscillations the expansion basis is different, i.e., polynomials instead of trigonometric functions, resulting in a different oscillatory behavior. This is illustrated in fig. 3, where (9) is numerically integrated by computing the spatial derivative spectrally. Because of insufficient resolution, i.e., a truncated Fourier representation, small over-and under-shoots develop. Unlike the dispersive oscillation in fig. 2, the Gibbs oscillation is symmetric.

Similar to the under- and over-shoots of the numerical solution of (9) in fig. 2, dispersion errors can cause erroneous scalar excursions in LES. In spite of the expectation (8) that the LES scalar field is bounded by its boundary values, in practice, this constraint is violated (e.g., Cook et al., 2004; Glaze, 2006; Matheou et al., 2010; Mattner, 2011; Subbareddy et al., 2014; Kartha et al., 2015). The relation between dispersive errors and boundedness for scalar conservation laws is addressed in numerous studies (e.g., Zhang and Shu, 2010; Verma et al., 2014), especially in the areas of gas dynamics (weak solutions of the compressible Euler equations), (e.g., Smolarkiewicz 
and Szmelter, 2009) and Reynolds Averaged Navier-Stokes (RANS) models (e.g., Williamson and Rasch, 1989; Shchepetkin and McWilliams, 1998; Smolarkiewicz and Szmelter, 2005; Nadukandi et al., 2010).

In contrast, references to the relation of dispersive errors and scalar boundedness in LES are rare (Glaze, 2006; Matheou et al., 2011). In addition, in LES, the SGS model provides a strong dissipation mechanism, which should ensure that flow fields remain adequately smooth. Typically LES numerical integrations do not require any additional or artificial dissipation mechanism for stabilization when a physical SGS model is used.

The preceding arguments lead to the hypothesis for the cause of scalar excursions in large-eddy simulations: Unphysical scalar excursions result from dispersive oscillations of the convection-term discretization at times and locations where the subgrid-scale model provides insufficient dissipation to produce a sufficiently smooth scalar field. According to this hypothesis, unphysical excursion locations need not correspond to locations of the highest scalar gradients, in contrast to numerical solutions of systems that can support discontinuities such as the Euler or Burgers' equations. In $\S 4$ we provide evidence in support of this hypothesis. In addition to the question of what causes the excursions, we also investigate the extent of the problem, define metrics for excursion diagnosis, and discuss implications for SGS modeling.

The problem of unphysical excursions is often managed by introducing numerical dissipation. Thus, in the present LES runs, two dissipative finite-difference schemes are also assessed, the

Table 1: Summary of convection schemes. The columns correspond to the legend of the scalar convection scheme used in fig. 10, the scalar and momentum convection schemes, and the corresponding convection-scheme references.

\begin{tabular}{|c|c|c|c|}
\hline Symbol & Scalar & Momentum & Comments and reference \\
\hline 2 & \multirow{3}{*}{\multicolumn{2}{|c|}{$\begin{array}{l}\text { 2nd order, fully conservative } \\
\text { 4th order, fully conservative } \\
6 \text { th order, fully conservative }\end{array}$}} & \multirow{3}{*}{ Non-dissipative; Morinishi et al. (1998) } \\
\hline 4 & & & \\
\hline 6 & & & \\
\hline Q & QUICK & 4 th order & Linear unwinding, somewhat dissipative; Leonard (1979) \\
\hline $\mathrm{S}$ & \multicolumn{2}{|c|}{ Pseudo-spectral } & Highest order of approximation; Chung and Matheou (2012) \\
\hline $\mathrm{M}$ & MC flux-limited & 4 th order & Monotonicity preserving, most dissipative; Van Leer (1977) \\
\hline
\end{tabular}


Quadratic Upstream Interpolation for Convective Kinematics (QUICK, Leonard, 1979) and a fluxlimited monotone scheme with the monotonized central (MC) limiter (Van Leer, 1977). QUICK is a linear upwinding scheme that is dissipative but does not guarantee monotonicity of convected fields, while the MC-limited scheme is dissipative and monotonicity preserving.

For all finite-difference simulations, momentum convection is always non-dissipative. Dissipative schemes are only used for scalar convection. For instance, for runs that are labeled "fourth-order", the momentum and scalar convection is fourth-order fully conservative; for runs that are labeled "monotone" the momentum convection is fourth-order fully conservative and the scalar convection is approximated with the flux-limited monotone scheme.

The main emphasis of the LES results and discussion is on the finite-difference approximations because they are most commonly used in applications. For reference, a pseudo-spectral (Rogallo, 1981) method is also used because it represents the highest-order numerical approximation for a given grid. Table 1 summarizes the convection schemes examined and compared.

In addition to the Eulerian finite-differences schemes presently employed, depending on the application, several alternative types of convection schemes have been proposed to maintain scalar boundedness, including, semi-Lagrangian formulations (e.g., Purnell, 1976; Staniforth and Côté, 1991), particle methods (e.g., Monaghan, 1988; Yoon et al., 1999), and weighted essentially nonoscillatory (WENO) schemes (Liu et al., 1994; Jiang and Shu, 1996). Further discussion of the various numerical techniques and references can be found, for instance, in Toro (1999) and LeVeque (2002).

\section{$2.3 \quad$ Flow-field resolution}

We briefly comment on the definition of grid resolution used in this work. Grid resolution is currently measured by the number of physical space grid points $N$ or, equivalently, the grid spacing, $\Delta x=L / N$, where $L$ is the computational domain length. For instance, simulations labeled $N=256$ correspond to a $256^{3}$ grid. Although this is a straightforward and commonly used measure, it is not necessarily the most relevant in comparisons involving different numerical schemes. This is because resolving power varies between different numerical approximations (e.g., see fig. 1). Thus, 
one could choose to vary grid resolution to keep the effective flow resolution constant. Another parameter is computational cost, which is a key consideration in practical applications. There are significant differences in the computational cost between the various numerical schemes. Therefore, another option is to keep the computational cost constant and vary grid-resolution accordingly.

\subsection{Subgrid-scale models}

The SGS model plays an important role in controlling the quality of the LES prediction. Unphysical scalar excursions are expected to exhibit a strong dependence on the SGS model and its parameters. Two SGS models are considered here, the constant-coefficient Smagorinsky (1963) and the stretched-vortex model (Misra and Pullin, 1997; Pullin, 2000; Voelkl et al., 2000). The two SGS models differ in their formulation and complexity.

\subsubsection{Smagorinsky}

The Smagorinsky model relies on an eddy-diffusivity closure. The SGS stress tensor and scalar flux are given by

$$
\begin{aligned}
\tau_{i j} & =-2 \nu_{t} \bar{S}_{i j}, \\
\sigma_{i} & =-\frac{\nu_{t}}{\mathrm{Sc}_{t}} \frac{\partial \bar{Z}}{\partial x_{i}},
\end{aligned}
$$

where $\bar{S}_{i j}=\left(\partial \bar{u}_{i} / \partial x_{j}+\partial \bar{u}_{j} / \partial x_{i}\right) / 2$, is the resolved-scale rate-of-strain tensor, $\mathrm{Sc}_{t}=0.33$ is the model turbulent Schmidt number, and

$$
\nu_{t}=\Delta^{2}\left(2 \bar{S}_{i j} \bar{S}_{i j}\right)^{1 / 2}
$$

is the model turbulent viscosity, $\Delta=C_{s} \Delta x$ is the characteristic SGS eddy length scale, which is related to the uniform grid spacing $\Delta x$ by the Smagorinsky constant $C_{s}$. Two values of $C_{s}$ are used in the present runs: $C_{s}=0.18$ and 0.23 . The former value is exact for homogeneous 
isotropic turbulence (e.g., Lesieur and Metais, 1996), while the latter is commonly used in LES of atmospheric flows (e.g., Beare et al., 2006; Matheou et al., 2011).

\subsubsection{Stretched-vortex model}

The stretched-vortex model relies on turbulence flow physics concepts, considering the turbulent region as an ensemble of vortex filaments with their own dynamical statistics. The forms of the SGS stress and scalar flux are

$$
\begin{aligned}
\tau_{i j} & =\left(\delta_{i j}-e_{i}^{v} e_{j}^{v}\right) K, \\
\sigma_{i} & =-\frac{1}{2} \Delta K^{1 / 2}\left(\delta_{i j}-e_{i}^{v} e_{j}^{v}\right) \frac{\partial \bar{Z}}{\partial x_{j}},
\end{aligned}
$$

where $K$ is the SGS turbulent kinetic energy (TKE), $\Delta$ is the filter-cutoff width, also identified as the SGS eddy length scale, $\partial \bar{Z} / \partial x_{j}$ is the resolved scalar gradient, and $e_{i}^{v}$ is the unit vector aligned with the SGS vortex. The basic form of the model, Eq. (12), originates from the expression for the SGS stress tensor in a homogeneous anisotropic turbulent flow consisting of a collection of straight axisymmetric vortex structures (Pullin and Saffman, 1994; Pullin, 2000).

Typically, a single orientation for SGS vortical structures is used for each grid cell. The most often used orientation alignment is with the most extensional eigenvector, $e_{3}$, of the resolved strainrate tensor, $\bar{S}_{i j}$ (Voelkl et al., 2000; Pantano et al., 2008), which is also employed in this study.

The SGS turbulent kinetic energy, $K$, is determined by integrating the modeled unresolved kinetic energy spectrum, $E(k)$, i.e.,

$$
K=\int_{\pi / \Delta}^{\infty} E(k) \mathrm{d} k
$$

associated with such an SGS field of stretched vortices. Even though (13) implies a sharp cutoff spectral filter, the LES filtering operation is formal and never employed; it merely indicates that scales smaller than $\Delta$ are to be treated as subgrid and modeled, regardless of whether or not they are resolvable by the grid. Two values of $\Delta$ are currently used, $\Delta=\Delta x$ (which is typically used) 
297

and $\Delta=2 \Delta x$, see also discussion in Chung and Matheou (2014) regarding the effects of variable cutoff scale on flow statistics and convergence.

The vortical structures are Lundgren (1982) spiral vortices with a three-dimensional energy spectrum given by

$$
E\left(k ; \lambda_{v}\right)=\mathcal{K}_{0} \varepsilon^{2 / 3} k^{-5 / 3} \exp \left[-k^{2} \lambda_{v}^{2}\right],
$$

where $\lambda_{v} \equiv\left[2 \nu /\left(3\left|\bar{S}_{v}\right|\right)\right]$ (Pullin, 2000), $\mathcal{K}_{0}$ is the Kolmogorov prefactor, $\varepsilon$ the local cell-averaged dissipation rate, $\nu$ the kinematic viscosity of the fluid, and

$$
S_{v} \equiv-\left(\delta_{i j}-e_{i}^{v} e_{j}^{v}\right) \partial \bar{u}_{i} / \partial x_{j}
$$

is the strain aligned in the ensemble SGS vortex axis.

The final step in determining the expressions for the subgrid terms is to estimate the product $\mathcal{K}_{0} \varepsilon^{2 / 3}$. This provides closure and determines the value of the subgrid kinetic energy using the local, resolved-scale, second-order velocity structure function $\bar{F}_{2}(r ; \mathbf{x})$ (Metais and Lesieur, 1992; Voelkl et al., 2000),

$$
\mathcal{K}_{0} \varepsilon^{2 / 3}=\frac{\bar{F}_{2}}{A \Delta^{2 / 3}}
$$

where

$$
A=4 \int_{0}^{\pi} s^{-5 / 3}\left(1-\frac{\sin s}{s}\right) \mathrm{d} s \approx 1.90695 .
$$

A local seven-point discrete approximation to a spherical average is used to estimate $\bar{F}_{2}$,

$$
\bar{F}_{2}(\Delta ; \mathbf{x})=\frac{1}{6} \sum_{j=1}^{3}\left[\left(\delta \bar{u}_{1}^{+}\right)^{2}+\left(\delta \bar{u}_{2}^{+}\right)^{2}+\left(\delta \bar{u}_{3}^{+}\right)^{2}+\left(\delta \bar{u}_{1}^{-}\right)^{2}+\left(\delta \bar{u}_{2}^{-}\right)^{2}+\left(\delta \bar{u}_{3}^{-}\right)^{2}\right]_{j},
$$

where

$$
\left(\delta \bar{u}_{i}^{ \pm}\right)_{j}=\bar{u}_{i}\left(\mathbf{x} \pm \Delta x_{j}\right)-\bar{u}_{i}(\mathbf{x})
$$

is the velocity difference of the component $u_{i}$ in the direction $x_{j}$ at $\mathbf{x}$. This allows the SGS terms to be estimated dynamically using only local instantaneous resolved fields without any temporal or spatial averages. 
All operations needed to evaluate the SGS terms are local in space and time, and the model is dynamic with no free parameters.

\subsection{Kinetic energy and scalar dissipation rates}

The Smagorinsky and stretched-vortex are equilibrium models, i.e., assume an approximate balance between turbulent production and dissipation within the volume of the grid cell and time step interval. Thus, the SGS kinetic energy and scalar variance dissipation rates can be estimated by,

$$
\varepsilon=-\tau_{i j} \bar{S}_{i j}
$$

and

$$
\varepsilon_{Z}=-\sigma_{j} \frac{\partial \bar{Z}}{\partial x_{j}}
$$

respectively. In a high Reynolds number flow, the resolved-scale dissipation rates are negligible and the SGS dissipation rates (20) and (21) are presently identified with the total dissipation.

In general, the stretched-vortex model allows for backscatter. However, for vortex alignment with the most extensional eigenvector of the resolved rate of strain tensor in a divergence-free flow the model becomes purely dissipative. Substituting the expression of the stress tensor in (20):

$$
\varepsilon=-\bar{S}_{i i} K+\lambda_{3} e_{i} e_{i} K
$$

where $\lambda_{3} \geq 0$ is the eigenvalue corresponding to $e_{3}$. The first term of (22) is zero because of (7a) and the second is positive definite, thus $\varepsilon>0$. The same holds for the Smagorinsky model since $\varepsilon=2 \nu_{t} \bar{S}_{i j} \bar{S}_{i j}>0$

\subsection{Numerical integration}

The finite-difference code integrates (7) on an Arakawa C (staggered) grid (Harlow and Welch, 1965; Arakawa and Lamb, 1977). An exact Poisson solver using discrete Fourier transforms is used 
to compute the modified pressure, $p^{\prime}$, in (7b), and satisfy incompressibility (Schumann, 1985). The semi-discrete system of equations is advanced in time using the third-order Runge-Kutta of Spalart et al. (1991). For all cases, second-order centered differences are used to approximate the spatial derivatives of the resolved viscous terms and SGS model. The LES code was successfully used in several previous studies (Matheou et al., 2011; Inoue et al., 2014; Matheou and Chung, 2014; Matheou and Bowman, 2016) and has been rigorously tested and validated.

The pseudo-spectral code is identical to the one used in Chung and Matheou (2012). Spatial derivatives are computed spectrally and nonlinear terms are evaluated in physical space and then transformed to Fourier space to advance the Fourier coefficients of the velocity and scalar fields in time. The integrating factor is used for the integration of the resolved-scale viscous terms. The same Runge-Kutta method as in the finite-difference code is used for time-marching. The $2 / 3$ rule (Orszag, 1971) is used for dealiasing, which implies that for a physical grid of length $N$, there are $N / 2+1$ independent Fourier coefficients, of which the $2 / 3$ are dealiased.

\subsection{Flow configuration, and initial and boundary conditions}

Scalar excursions are studied in a temporally evolving shear flow. The flow configuration chosen is simple enough for a fundamental study but, at the same time, exhibits local characteristics observed in more complex flows. We consider an infinite stack of vertical layers with period $L=4 \pi \mathrm{m}$, a spatially alternating initial velocity field of $u= \pm 1 \mathrm{~ms}^{-1}$, and initial freestream scalar values $Z=0,1$ (fig. 4). The computational domain is a cube with sides of length $L$ with triply periodic boundary conditions. The flow is within the applicability of the incompressible numerical method used in this discussion, while achieving a high Reynolds number. Domain periodicity precludes any effects of discrete boundary conditions, or commutation errors from affecting the results.

The streamwise, cross-stream, and transverse directions and components are denoted by $(x, y, z)$ and $(u, v, w)$, respectively. The initial condition for the velocity field is

$$
u_{i}(t=0, x, y, z)=u_{i}^{\prime}+\partial p^{\prime} / \partial x_{i}
$$

where $p^{\prime}$ is the Lagrange multiplier that imposes the incompressibility constraint on the initial 
velocity field, and

$$
\begin{aligned}
& u^{\prime}(t=0, x, y, z)=f(z)+a(z)\left(\sin 4 x \cos y+r_{u}(x, y, z)\right) \\
& v^{\prime}(t=0, x, y, z)=a(z)\left(\cos 8 x \sin y+r_{v}(x, y, z)\right) \\
& w^{\prime}(t=0, x, y, z)=a(z)\left(\sin 2 x \cos 4 y+r_{w}(x, y, z)\right)
\end{aligned}
$$

where $r$ is a random number in $[-0.5,0.5]$,

$$
f(z)=\tanh (40 \operatorname{modulo}(z, 4 \pi)-2 \pi)-\tanh (40 \operatorname{modulo}(z, 4 \pi)-4 \pi)-\tanh (40 \operatorname{modulo}(z, 4 \pi)),
$$

and

$$
a(z)=0.1 \exp \left[-100(\operatorname{modulo}(z+\pi, 2 \pi)-\pi)^{2}\right]
$$

The initial condition for the passive scalar is

$$
Z(t=0, x, y, z)=(1+f(z)) / 2 .
$$

The initial field is chosen to excite both spanwise and streamwise instabilities, such that the resulting shear layers acquire significant three-dimensional structure. A visualization of the development of the shear instabilities is shown in fig. 4. The top plane of the computational domain corresponds to a middle plane of the mixing zone.

For the current simulations, the Schmidt number is set to unity, approximating gas-phase diffusion, and $\nu=1 \times 10^{-5} \mathrm{~m}^{2} \mathrm{~s}^{-1}$.

Figure 4 shows the flow evolution, including the initial development of the Kelvin-Helmholtz instability, the transition to fully-developed turbulence and the amalgamation of the large-scale structures to a three-dimensional mixing zone. The flow attains a Reynolds number based on the width, $\delta$, of the mixing zone of about $5 \times 10^{5}$, well in excess of the mixing transition threshold (Dimotakis, 2000). The mixing zone width, $\delta$, is defined as the $1 \%$ thickness, i.e. $0.01 \leq\langle\bar{Z}\rangle \leq 0.99$. Angle brackets $\langle\bullet\rangle$ denote a horizontal average. 


\section{Passive scalar excursions}

Two types of diagnostic metrics of invalid scalar values are considered: global excursions that violate the scalar boundary values and a local constraint. The two diagnostics have different properties and implications for LES but also exhibit some overlap. Accordingly, the naming of the two types of metrics is intended primarily for distinguishing between the two.

\subsection{Global or persistent excursions}

The definition of a global (unphysical) excursion is straightforward: any scalar value outside the bounds of the initial and boundary conditions. In the present LES runs, any value $\bar{Z}<0$ or $\bar{Z}>1$ is a global (unphysical) excursion. Because this type of excursion or model error is defined with respect to a global attribute and holds for all times, it can be characterized as a global or persistent error. That is, a value of $\bar{Z}=-0.1$, which can correspond to a species mixing ratio, is invalid at any location (global) at any time (persistent). Global excursions are the most significant source of error since they are unphysical and must be specially treated when used as input for physical processes, for instance, thermodynamic and reaction-rate calculations. Therefore, most of the results in the present discussion pertain to global excursions.

\subsection{Local or internal excursions}

If erroneous scalar values that violate global bounds can be created during the numerical integration, we may expect that the same process can yield erroneous values even inside the range $0<\bar{Z}<1$ that cannot be detected by the previous global criterion. These excursions are local or internal in character, since they occur in the mixed fluid (internal) and for their identification the local flow field must be considered.

In order to define a local excursion, the scalar transport equation is integrated in time from an initial state, $t$, for a time interval $\Delta t$,

$$
\int_{t}^{t+\Delta t} \frac{\partial Z}{\partial t} \mathrm{~d} t+\int_{t}^{t+\Delta t} \frac{\partial u_{j} Z}{\partial x_{j}} \mathrm{~d} t=\mathcal{D} \int_{t}^{t+\Delta t} \frac{\partial^{2} Z}{\partial x_{i}^{2}} \mathrm{~d} t
$$


The value of $Z$ at $t+\Delta t$ depends on contributions to $Z_{t}$ from convection and diffusion,

$$
Z_{t+\Delta t}=Z_{t}-\int_{t}^{t+\Delta t} \frac{\partial u_{j} Z}{\partial x_{j}} \mathrm{~d} t+\mathcal{D} \int_{t}^{t+\Delta t} \frac{\partial^{2} Z}{\partial x_{i}^{2}} \mathrm{~d} t
$$

In the limit of vanishing positive diffusivity, $\mathcal{D} \rightarrow 0^{+}$, the sign of the diffusive term determines the relation of $Z_{t+\Delta t}$ with respect to the inviscid limit. Thus, the local constraint of the Eulerian $Z$ is

$$
Z_{t+\Delta t}>\operatorname{sgn}\left(\int_{t}^{t+\Delta t} \frac{\partial^{2} Z}{\partial x_{i}^{2}} \mathrm{~d} t\right)\left(Z_{t}-\int_{t}^{t+\Delta t} \frac{\partial u_{j} Z}{\partial x_{j}} \mathrm{~d} t\right) \equiv Z_{\Delta t, \mathrm{inv}}
$$

This constraint is local in space and time, and it is a result of the requirement that only local mixing/diffusion of $Z$ is allowed. That is, it requires that local $Z$ values be on the "convex side" of the inviscid limit. Accordingly, referring to dispersive oscillations of fig. 2, only half of the oscillation pattern violates the local excursion criterion, since the rest is on the "convex side" of the inviscid limit. Both global and local criteria diagnose unphysical scalar excursions and not all sources of error in LES.

To diagnose (30) in LES, the convection term must be computed sufficiently accurately for an interval $\Delta t$, which requires performing an inviscid integration. A more-accurate inviscid integration for the scalar is performed on a finer grid. In the present runs, we utilize a refinement factor of 4 , $\Delta x_{\text {fine }}=\Delta x / 4$, for diagnostic purposes. At time $t$, the velocity and scalar fields are interpolated on the fine grid using trilinear interpolation, which preserves total mass. The scalar equation on the fine grid is then integrated for time $\Delta t$ using the current fine-grid-interpolated velocity field at each sub-step of the Runge-Kutta. This process results in the RHS of (30), $Z_{\Delta t \text {,inv }}$. The constraint (30) is then evaluated by comparing the co-located $Z_{\Delta t \text {,inv }}$ on the fine grid and $\bar{Z}$ on the original coarse LES grid. No averaging is performed from fine to coarse grids, because this would result in diffusion of the fine-grid $Z$ and invalidate the inviscid nature of the integration that is required.

To diagnose local excursions using (30), the integration must be inviscid as noted above, which implies a sufficiently small $\Delta t$ and a sufficiently high refinement factor such that $Z_{\text {fine }}$ is wellresolved. As time progresses, $Z_{\text {fine }}$ develops smaller scales because of the scalar-variance cascade. In practice, up to 10 time steps for $\mathrm{CFL}=1.2$ of the fine grid were used without observing grid-scale 
fluctuations on the fine grid. The results of $\S 4$ are for $\Delta t$ equal to one time step of the fine grid.

Finally, we reiterate that the local criterion identifies unphysical excursions at time $t+\Delta t$ with respect to the scalar field at $t$, whereas the global criterion identifies unphysical values that could have been created at any previous time instance.

\section{Results}

\subsection{Control for flow evolution}

Before considering the statistics of scalar excursions, we examine the characteristics of the turbulent flow field. The aim of the current simulations is to isolate the effects of numerics and SGS closure with respect to unphysical scalar excursions, while keeping all other flow and simulation aspects identical, so as to have a meaningful comparison of the scalar statistics for different schemes and SGS models.

Figure 5 shows the evolution of the mixing zone width $\delta$. Figure 6 shows a higher-order statistic, the vertical distribution of the resolved-scale scalar variance, $(\bar{Z}-\langle\bar{Z}\rangle)^{2}$, at $t=20$ s. Figures 7 and 8 show scalar dissipation rates. Figure 7 shows dissipation rates for $N=256$, and $C_{s}=0.18$ and 0.23 for the Smagorinsky model and $\Delta=\Delta x$ for the stretched-vortex model. Figure 8 shows scalar dissipation for three runs with fourth order convection and the stretched-vortex model for two resolutions $(N=256$ and 512$)$ and two cutoff scales $(\Delta=\Delta x$ and $2 \Delta x)$. Figure 7 only shows the physical scalar dissipation (21) and does not include the numerical dissipation in the cases of the QUICK and monotone schemes. For these convection schemes, the physical dissipation rate in fig. 7 is less compared to the non-dissipative schemes since a significant fraction of the scalar variance dissipation is furnished by the numerics in those cases.

All flow statistics show negligible differences between the finite-differences numerical schemes, grid resolution, and SGS models. The results of the spectral LES using the stretched-vortex model exhibit deviations from the finite difference schemes that are likely because of discretization effects of the SGS terms and are not related to scalar excursions. For the finite differences schemes most differences in figs. 5-7 are observed between the two SGS models. The Smagorinsky model 
produces a somewhat wider mixing zone and larger scalar dissipation values. The differences in fig. 6 are mostly attributable to turbulent flow variations and the lack of statistical convergence with averaging that has been performed for only a single flow snapshot.

At first instance, the small differences in fig. 5 and 6 may be unexpected. However the differences are small because, $(a)$ momentum convection is always non-dissipative and the scalar is passive and, (b) the flow is well-resolved, thus both SGS models perform well. The ratio of resolved to total turbulent kinetic energy (TKE) can be used as a criterion for convergence, and for the coarsest runs, $N=256$, this ratio is always more than $90 \%$, which is more than the proposed required $80 \%$ (Pope, 2004; Matheou et al., 2010).

For Smagorinsky-type models, the grid-scale Reynolds number, $\operatorname{Re}_{\Delta x} \equiv \Delta u \delta /\left\langle\nu_{t}\right\rangle$, can be used to assess grid-resolution and the adequacy of scale separation in the LES. The mean value of the turbulent viscosity is estimated by $\left\langle\nu_{t}\right\rangle=C_{k} \Delta x q^{1 / 2}$ (Moeng and Wyngaard, 1988), where $q \equiv\left\langle\bar{u}_{i} \bar{u}_{i}\right\rangle / 2$ is the resolved-scale TKE and $C_{k}=C_{s}^{4 / 3} C_{\varepsilon}^{1 / 3}$ a dimensionless constant that relates the Smagorinsky and dissipation constants $\langle\varepsilon\rangle=C_{\varepsilon} q^{3 / 2} / \Delta x$, as originally proposed by Taylor (1935). Using $C_{\varepsilon} \approx 0.93$ (Lilly, 1967) and the value of TKE in the mid plane of the shear layer, $q=0.09 \mathrm{~m}^{2} \mathrm{~s}^{-2}, \operatorname{Re}_{\Delta x}=4.3 \times 10^{3} \gg 1$ for $N=256$ and $C_{s}=0.23$, which is a relatively high value. As implied by figs. 5 and 6 , the TKE is the same for all LES runs.

The present numerical experiments are designed to produce statistically identical turbulent flows such that scalar excursions can be studied while controlling for flow geometry, and it should not be inferred from these results that controlling scalar excursions represents the sole criterion for simulation fidelity, or that the numerical scheme and SGS model do not affect LES predictions.

\subsection{Global-excursion statistics}

Two types of global scalar excursion statistics are computed: the point-wise minimum and maximum of the passive scalar in the entire domain and the volume fraction of fluid with $\bar{Z}$ values greater/lower than a given threshold value. Figure 9 shows examples of these statistics as a function of time for a run with the fourth-order scheme and the stretched-vortex model for $N=256$ grid. The left panel of fig. 9 shows the magnitude of under- and over-shoots, i.e. $-\min (\bar{Z})$ and 
$\max (\bar{Z})-1$. In the current study, we will refer to the magnitude of under- and over-shoots with the term excursion, without making a distinction between unphysical under- and over-shoots. The right panel shows the volume fraction of fluid that exceeds a $5 \%$ excursion (in absolute value), i.e., $\bar{Z}<0.05$ and $\bar{Z}>1.05$. Five excursion thresholds are explored, at 1, 2, 5, 10, and $20 \%$.

To aid in the comparison of different schemes, scalar statistics are further reduced by averaging in time and averaging the under- and over-shoots. This procedure produces a single value for each statistic. Time averaging is performed between $t=10-20 \mathrm{~s}$, an interval chosen because the flow is well developed with multi-scale turbulent structures, but with a significant fraction of fluid as yet unmixed. The lowest row of fig. 4 shows the flow instances at the beginning and end of the averaging interval. Scalar excursion averages are denoted by black horizontal lines in the panels of fig. 9 .

A three-dimensional parameter space is studied with variables of grid resolution, convection scheme, and SGS model type (which includes SGS model parameters). Overall, 37 LES runs were carried out. Two grid resolutions $N=256$ and 512 are mainly used. One run with the stretchedvortex model is carried out at $N=1024$ to investigate the distribution of scalar gradients on resolution. A total of six scalar convection schemes are used. Their characteristics are documented in $\S 2.2$. Two SGS models are used, the constant-coefficient Smagorinsky and stretched-vortex model, and two sets of runs are carried out for each by varying a model parameter. Two values of the Smagorinsky constant are used, $C_{s}=0.18$ and 0.23 ; and two values of the cutoff scale $\Delta=\Delta x$ and $2 \Delta x$ are used for the stretched-vortex model.

For all finite-difference runs, momentum convection is non-dissipative (see Table 1) and the fourth-order momentum convection scheme is used when QUICK and the MC flux-limited schemes are used for the scalar. Because the initial condition is identical for all runs, simulations with the fourth-order, QUICK, and monotone scalar convection schemes have the same velocity field.

In accord with the hypothesis that scalar excursions are caused by dispersion errors of the spatial derivative approximation, aspects of time integration and resolved-scale viscous terms are not included in the parameter study. For completeness, these effects are investigated and documented in the Appendices. Perhaps as expected, time-step size and resolved-scale viscous terms have no 
effect on scalar excursions.

Figure 10 shows the global scalar excursion statistics for all LES runs. Several general observations can be made. Both the numerical scheme and SGS model have a significant influence on scalar excursion statistics. The largest excursions are observed for the lowest-order convection scheme and improve (become progressively smaller) as the order of accuracy is increased. QUICK appears to further reduce excursions while the pseudo-spectral code produces even smaller excursions. As expected, the monotone scheme preserves the boundedness of the passive scalar, essentially by construction. This ranking of convection schemes holds when both the maximum excursion is considered and for volume-fraction statistics. Runs with the stretched-vortex model produce larger small-scale fluctuations because the SGS model is in the mean less dissipative than the Smagorinsky, fig. 7.

\subsubsection{Resolving bandwidth and numerical dissipation}

Global scalar excursions become smaller as the order of accuracy is increased. For all statistics depicted in fig. 10, the rate of improvement decreases as the order of accuracy increases, consistently with the improvement in bandwidth in fig. 1. The benefit of a higher-order non-dissipative scheme is greater for larger excursions since the volume fraction of excursions over $5 \%$ decreases significantly when the order of accuracy is increased from second to sixth. Considering the results of the spectral code as a limiting case for the non-dissipative finite differences, we can infer that scalar excursions do not converge to zero as the order of accuracy approaches a spectral approximation and that some residual error should be expected. The spectral run exhibits small-amplitude oscillations from small aliasing errors, similar to the Gibbs under- and over-shoots of fig. 3, and not from dispersion errors since the approximation is non-dispersive. The spectral approximation error is very small and only registers in the maximum excursion and volume fraction $<5 \%$ metrics.

\subsubsection{Subgrid-scale model}

Overall, the stretched-vortex model exhibits larger peak excursions and larger volume fractions compared to the Smagorinsky. This is expected, since the Smagorinsky model is more dissipative 
and produces smoother flow fields. In addition, the performance of the Smagorinsky model depends on the choice of the constant. For instance, a reduction of the Smagorinsky model constant from $C_{s}=0.23$ to 0.18 , which decreases the SGS model dissipation, results in excursion statistics similar to those with the stretched-vortex model.

The greatest reduction in global excursions is achieved when $\Delta=2 \Delta x$ in runs with the stretched-vortex model. These runs have $N=512$ grids. However, the effective resolution is comparable to $N=256$. Although this entails a significant computational expense - a factor of 16 - for a similarly resolved flow range of scales, the global excursion error is significantly reduced with a negligible volume fraction of $>5 \%$ for global excursions. Moreover, the non-dissipative fourthand sixth-order schemes perform better in this case than the dissipative QUICK scheme.

\subsubsection{Grid resolution}

Two main sets of runs are carried out using $256^{3}$ (denoted as $\left.N=256\right)$ and $512^{3}(N=512)$ grid points. The maximum excursion and volume fraction show opposite trends with respect to resolution. Maximum excursions increase as resolution increases, whereas associated volume fractions decrease. The reason for the increase in the maximum excursion is statistical and not traceable to numerics or flow modeling. Because the maximum excursion considers the value of a grid-wise point quantity, i.e., a voxel and not an average, it depends on the number of grid points, i.e., the sample size. For the finer grid, the sample size increases 16 -fold ( 8 times more grid points and 2 times more time steps), therefore, the probability of a single higher extreme scalar value is higher.

In contrast, the volume fraction of unphysical excursions decreases with resolution because the SGS model performs better. Less energy (kinetic for the velocity field and scalar variance) remains unresolved as the grid becomes finer and the two models used here can better capture the SGS flow and predict the space-time local dissipation to reduce dispersion errors leading to unphysical excursions. However, as noted in $§ 2.1$, as resolution increases, and as long as the LES is far from being a DNS, the flow field does not become "smoother" and any gain in accuracy of modeling is because of expected improvements in SGS model performance. The SGS model improvement can be 
a direct result of weaker SGS fluctuations without any gains in the fidelity of representation of the unresolved turbulence. We use the expression "expected improvement" because not all SGS models will necessarily statistically converge monotonically to fully resolved flow. As corroborated by the current results, the stretched-vortex and Smagorinsky models perform better as grid resolution is increased. Similarly to what is observed with the increase in order of accuracy, increases in resolution have a greater impact on the largest excursions.

Figure 11 supports these arguments and shows the probability density function (p.d.f.) of the normalized scalar gradient at three resolutions, $N=256,512$, and 1024, with the fourth-order scheme and stretched-vortex model. The discrete approximation, $\Delta_{i}^{\alpha} Z / \Delta^{\alpha} x_{i}$ of the scalar gradient, $\partial Z / \partial x_{i}$, where $\alpha$ denotes the corresponding difference operator of order $\alpha$, is multiplied by $\Delta^{\alpha} x_{i}$ to remove the dependence on grid size. That is, fig. 11 shows the p.d.f. of the scalar differences, $\Delta_{i}^{\alpha} Z$, in the entire domain in the $t=10-20 \mathrm{~s}$ interval for each direction. Although scalar gradients increase as grid resolution increases in the LES, the increase in resolution is slightly faster and the grid-scale scalar differences decrease as resolution increases. However, because sample size increases with resolution, the tails of the p.d.f. extend to higher absolute values of the scalar difference.

Even though the current results show that all LES solutions overall improve with increasing resolution and the maximum and volume fraction of the excursion can be understood, it is troublesome that the maximum excursion increases with increasing resolution and exhibits values much higher that the one-dimensional convection dispersive excursions (fig. 2). It is likely that some error compounding is occurring particularly for the excursions that "wash out" in the free stream where no active dissipation mechanism is present. Based on the present LES runs, we cannot conclude if the maximum excursion increasing trend will reverse when the resolution is high enough to resolve the turbulent flow, i.e., as LES approaches a DNS, or at a coarser resolution. Finally, we emphasize that the maximum excursion metric is not a well-defined error metric in LES because it is based on a single grid cell value and not a well-constructed flow statistic (Pope, 2004). 


\subsection{Local excursions}

Figure 12 shows instantaneous slices from the run with the fourth-order fully conservative scheme, stretched-vortex model, and $N=256$. The magnitude of the scalar gradient $\left[\left(\partial \bar{Z} / \partial x_{i}\right)^{2}\right]^{1 / 2}$ is plotted along with contours of the $1 \%$ excursions in the left column panels. The nature of dispersive oscillations is distinctly discernible in fig. 12. Oscillations occur in regions with diverse values of the scalar gradient and do not preferentially correlate with the highest values. Animations of the scalar gradient and excursions, similar the instantaneous images of fig. 12, were also studied to examine if the excursions corresponding to smaller gradients in the instantaneous images had been created by past larger gradients that were convected/diffused. Diffusion of scalar gradients with persisting scalar excursions was not observed, likely because scalar excursions are short-lived with time-scales comparable to grid-scale turbulent motions.

Figure 13 shows two horizontal slices from a run identical to the one shown in fig. 12, but with the modified second-order convection scheme (see $\S 2.2$ ). Similar to the dispersive oscillations in fig. 2, the side of some of the oscillations and scalar excursions with respect to mixed-fluid patches changes between figs. 12 and 13.

The panels of the right column of fig. 12 show the magnitude of the local excursions, $\max (0, Z-$ $\left.Z_{\Delta t \text {,inv }}\right)$. The local excursions are relatively small because they are excursions with respect to the local flow field of the previous time step. Most local unphysical excursions occur in the mixed fluid, are widespread, and mostly structureless. Figure 14 shows the volume fraction of local excursions that occur in the mixed fluid versus the total volume of excursions with respect to time. Two mixed-fluid thresholds are used, $0.1<\bar{Z}<0.99$ and $0.05<\bar{Z}<0.95$. Significantly, roughly $90 \%$ of the volume of unphysical local excursions are internal excursions, i.e., they do not violate global scalar boundedness constraints.

To help assess the relation between scalar gradient and local excursions, fig. 15 shows the frequency distribution (i.e., the two-dimensional histogram) of the local excursion-scalar gradient pairs for 11 flow realizations in the $t=10-20 \mathrm{~s}$ interval, for a run with $N=256$, fourth-order convection and the stretched-vortex model. Because excursions ( $y$-axis) are relatively rare, about $97 \%$ of the occurrences are accounted in the $y=0$ bins in the histograms of fig. 15: the dark band 
along the $x$-axis. The pairing/association of excursions to scalar gradient is done in two ways: using co-located pairs (shown in the left panel of fig. 15) and by pairing each excursion value with the maximum gradient magnitude in the surrounding $7 \times 7 \times 7$ grid cells (right panel). This is to account for the offset of the maximum dispersion error with respect to the local gradient, as shown in fig. 2, which is usually $2-3$ grid cells. The histograms in fig. 15 confirm the observation from fig. 12 that excursions and scalar gradient magnitude are not well correlated. Even when accounting for noncolocation, maximum excursions tend to occur at intermediate values of scalar-gradient magnitude. Moreover, for a given scalar excursion value, the gradient distribution is broad.

Figure 16 shows the frequency distribution of the local excursions and estimate of scalar dissipation rate (21). Unlike the local excursion-scalar gradient pairs of fig. 15, a significant fraction of local unphysical excursions occurs near grid points with low scalar dissipation values. There is a very small fraction of grid points with negative dissipation rates but for most of these points no excursions are observed (the contour scales in figs. 15 and 16 are logarithmic). The right panel of fig. 16 shows pairs of scalar excursions with the minimum dissipation in the surrounding $7 \times 7 \times 7$ grid cells (similar to the right panel of fig. 15). All grid cells with unphysical scalar excursions are at a distance less than 4 grid cells from locations with nearly zero scalar dissipation rate.

\section{Discussion}

The analysis of scalar-excursion statistics supports the main hypothesis that unphysical scalar excursions result from dispersive errors of the convection-term discretization at times and locations where the subgrid-scale model provides insufficient dissipation to produce a sufficiently smooth scalar field. This proposition has an important implication: dispersion errors (and consequently unphysical scalar excursions) do not necessarily occur where scalar gradients are highest since there can be instances where the SGS model supplies insufficient dissipation to smooth/regularize a moderate gradient. We note that perhaps because of adaptations of the study of numerical methods for the solution of differential equations (e.g., Euler or Burgers' equations) for LES (turbulence) modeling, it is commonly assumed that unphysical scalar excursions occur (in the vicinity) of the highest scalar gradients. Our findings do not support this supposition. 
In the current study, most of the analysis concerns passive scalar excursions in LES and compares different simulation parameters - numerical scheme, SGS model, and resolution - with respect to excursion statistics. However, scalar excursions are only one aspect of LES modeling and a successful simulation should take into account more aspects of the flow to be predicted. In other words, the results of fig. 10 may imply that a monotone scheme is the best choice. In practice, there is a tradeoff between conflicting requirements. The monotone scheme introduces artificial numerical dissipation that can have deleterious effects on LES predictions (e.g., Matheou et al., 2011). Alternatively, the fully conservative schemes have the desirable properties of preserving kinetic energy and scalar variance, which have been shown to benefit turbulence simulations (Morinishi et al., 1998), but numerical solutions can exhibit unphysical scalar excursions. Linear upwinding schemes, such as QUICK, present a tradeoff between no dissipation and non-linear monotonicity-preserving schemes by allowing small excursions $<5 \%$ without introducing excessive numerical dissipation.

The impact of numerical dissipation on the turbulent flow can be quantified by examining the scalar dissipation spectra. Figure 17 shows one-dimensional scalar spectra along the streamwise direction on the shear layer mid plane. Numerical dissipation decreases scalar fluctuations at high wavenumbers with differences of almost a decade in magnitude between the high-order nondissipative and monotone schemes. To better illustrate the effect of scalar-fluctuation dumping at the highest wavenumbers both scalar, $E_{Z Z}$, and scalar-gradient, $k_{x}^{2} E_{Z Z}$, spectra are shown in fig. 17. In the scalar spectra the integral of the area between dissipative and non-dissipative schemes corresponds to the amount of scalar variance that is destroyed by numerical dissipation. The current flow setup is designed to keep the flow nearly statistically identical for all runs. Differences between different schemes can be larger in other applications. Overall, the impact of artificial dissipation in LES is complex and its effects depend on the application and model details (e.g., Brown et al., 2000; Meinke et al., 2002; Park et al., 2004), see also Sagaut (2006, p. 299) and references therein. The distribution of local unphysical excursions (fig. 12) shows that the error is widespread. Even though this may be disconcerting, it is not surprising. The current results for unphysical excursions of passive scalar mixing in LES are consistent with error estimates for the velocity field (Ghosal, 1996). Similar to the analysis by Ghosal (1996) of LES errors for the velocity field, increasing 
the order of accuracy (bandwidth) of a finite-difference scheme results in only a slow reduction of differentiation error. In contrast, increasing the ratio of the cutoff scale to grid spacing, $\Delta / \Delta x>1$, while keeping $\Delta$ constant, is found to be an effective strategy to deduce unphysical scalar excursions for non-dissipative convection schemes, even though computationally costly for a given (effective) resolution. Again, this is qualitatively consistent with Ghosal's (1996) estimate for the rate of error reduction of $(\Delta x / \Delta)^{-3 / 4}$. Note that for passive scalars any errors in the velocity field, including dispersive oscillations of $u_{j}$, do not affect the scalar boundedness property (6) because $u_{j}$ is merely a coefficient in the linear equation for $Z$ (4).

\section{Conclusions}

The current investigation characterizes unphysical passive-scalar excursions in large-eddy simulations (LES), quantifies the numerical model error, and examines methods for diagnosis and assessment of the problem. The analysis of scalar-excursion statistics shows that unphysical scalar excursions in LES result from dispersive oscillations of the convection-term discretization at times and locations where the subgrid-scale model provides insufficient dissipation to produce a sufficiently smooth scalar field.

Scalar excursions are studied in a triply periodic shear flow arranged in a vertically periodic stack of mixing layers. In the LES runs, three parameters are varied: the discretization of the convection terms, grid resolution, and SGS model type, including model parameters. Two types of excursion diagnostics are studied: global excursions, which violate the boundary values of the scalar transport equation; and local excursions, which violate local scalar bounds. Global excursions are analyzed by considering the minimum and maximum in the entire computational domain and the volume of fluid with scalar values exceeding an excursion threshold. Local excursions are primarily used to obtain unphysical scalar-excursion information in the mixed fluid.

For a family of non-dissipative fully-conservative schemes (Morinishi et al., 1998), excursions decrease as the order of accuracy of the numerical approximation increases. However, the improvement rate decreases with increasing order of accuracy, and runs with a pseudo-spectral code indicate that scalar excursions need not converge to zero as the order of approximation approaches spec- 
tral. A Quadratic Upstream Interpolation for Convective Kinematics (QUICK, Leonard, 1979), a linear upwinding scheme, results in smaller excursions than non-dissipative schemes. A flux-limited monotone scheme is also assessed and, as expected, no scalar excursions are observed. However, in addition to scalar excursions, other simulation aspects should be considered in a practical LES, with the impact of numerical dissipation on the quality of flow prediction being only one. Scalar spectra show damping of the high wavenumbers when numerical dissipation is introduced. Although numerical dissipation is present in many computations, its effects and tradeoffs with respect to the overall LES prediction fidelity of scalar mixing is complex and remains largely unexplored.

As expected, unphysical scalar-excursion statistics are found to strongly depend on the SGS model and model parameters. Two SGS models were assessed, the stretched-vortex and constantcoefficient Smagorinsky. When the characteristic SGS length scale is equal to the grid spacing, the Smagorinsky model, which is more dissipative, results in smaller excursions, although the magnitude of the excursions is strongly dependent on the model constant. The excursions are significantly reduced when the characteristic SGS scale is set to double the grid spacing in runs with the stretched-vortex model, even though that requires a greater computational effort.

The maximum excursion and volume fraction show opposite trends with respect to resolution. The maximum excursion increases as resolution increases, whereas the volume fraction decreases, as might be expected because the number of grid points (sample size) increases with resolution. In contrast, the volume fraction of the excursions decreases with resolution because the SGS models perform better at higher grid resolutions.

The overall conclusion is that improvements in SGS models are required to address unphysical scalar excursions. The causes that lead to insufficient SGS scalar dissipation are currently not well understood. Moreover, it is not clear if the problem originates from the momentum SGS modeling or only affects the scalar fields, implying a breakdown in assumptions that relate the turbulent momentum and scalar fields. Even though the scalar excursion volumetric error is small, its nature can lead to error compounding in LES involving active scalars. Higher order non-dissipative finitedifference discretizations do not yield fast error reduction (but low-order discretizations produce excessively high errors) and general dissipative schemes must be regarded as a blunt tool, since they 
introduce artificial dissipation in locations where the SGS model may already provide the necessary dissipation.

\section{Acknowledgments}

This work was supported by AFOSR Grant No. FA9550-12-1-0461, DOE Grant No. DE-NA0002382, and the John K. Northrop Chair of the California Institute of Technology. The authors would also like to acknowledge discussions and collaborations with Prof. G. Candler and his research group at the University of Minnesota, and Prof. D. Meiron and Prof. D. Pullin at Caltech. We would like to thank Dr. D. Chung (University of Melbourne) for making available the pseudo-spectral code used in this study. This research was carried out at the California Institute of Technology and the Jet Propulsion Laboratory, California Institute of Technology, the latter under a contract with the National Aeronautics and Space Administration. 


\section{A Time integration and conservation verification}

Because scalar excursions resemble small-scale numerical instabilities, the verification of the RungeKutta time integration method and the fully-conservative convection schemes is documented in this appendix to exclude errors or instabilities in the numerics that can cause scalar excursions. A verification of the implementation of the convection discretization and the conservation properties can be carried out by tracking the evolution of the conserved quantities, $u_{i}^{2}$ and $Z^{2}$, in a simulation without any diffusive terms, i.e., a numerical integration of the incompressible Euler equations. The $u_{i}$ and $Z$ fields are initialized using random numbers and then $u_{i}$ is made divergence-free by applying the Lagrange multiplier (23). The two conserved quantities are computed on the grid,

$$
\mathcal{K}=\sum_{\operatorname{all}(i, j, k)} u(i, j, k)^{2}+v(i, j, k)^{2}+w(i, j, k)^{2}
$$

and

$$
\mathcal{V}=\sum_{\operatorname{all}(i, j, k)} Z(i, j, k)^{2}
$$

the kinetic energy and scalar variance, respectively. The sum is over all velocity (or scalar to form $\mathcal{V})$ squared values at their locations on the grid without any interpolation (see, Morinishi et al., 1998). The integration is performed with a constant time step $\Delta t$ up to time $T$. Ideally, $\mathcal{K}$ and

Table 2: Scalar variance and kinetic energy conservation error with respect to time step size. The error decreases with the expected rate of $\Delta t^{3}$, the truncation error of the Runge-Kutta time integration.

\begin{tabular}{|c|c|c|c|c|c|c|c|c|}
\multirow{3}{*}{$n$} & \multicolumn{1}{|c|}{$\Delta t_{n}$} & $\mathrm{CFL}_{n}$ & \multicolumn{2}{|c|}{$\log _{2}\left(E_{n} / E_{n-1}\right)$} \\
& & & Mom. order & Scalar & Mom. & Sth order & \multicolumn{2}{|c|}{ 6th order } \\
Mom. & Scalar \\
\hline 1 & 0.2 & 1.45 & - & - & - & - & - & - \\
2 & 0.1 & 0.72 & 2.6913 & 2.67943 & 2.62389 & 2.61074 & 2.5926 & 2.58051 \\
3 & 0.05 & 0.36 & 3.09928 & 3.09524 & 3.08549 & 3.08028 & 3.0784 & 3.07427 \\
4 & 0.025 & 0.18 & 2.99085 & 2.99034 & 2.98751 & 2.98709 & 2.98613 & 2.98574 \\
5 & 0.0125 & 0.09 & 2.9797 & 2.97978 & 2.9787 & 2.97899 & 2.97845 & 2.97864 \\
6 & 0.00625 & 0.04 & 3.00842 & 3.00833 & 3.00826 & 3.00817 & 3.00819 & 3.00813
\end{tabular}


$\mathcal{V}$ at $t=0$ and $t=T$ should be equal. However, a very small amount of dissipation is introduced by the time-marching method, since numerical stability requires that the amplitude of all modes is unchanged or damped. Dissipation is proportional to the truncation error of the third-order Runge-Kutta, in this case, $\sim \Delta t^{3}$. Therefore, by performing several simulations with variable time step $\Delta t$ and computing the error, $E(\Delta t)=\mathcal{K}(t=0)-\mathcal{K}(t=T)$, we expect the error to decrease proportionally to the order of accuracy of the time-marching scheme. Table 2 shows the errors for momentum and scalar for the three finite-difference schemes with respect to time-step size and verifies that the convection schemes in the current implementation are fully conservative.

As an additional check, a pair of LES runs was carried out with different CFL numbers. The $N=256$ grid, with the fourth-order scheme and stretched-vortex model was ran at CFL $=1.4$ and 0.1 (the Runge-Kutta CFL stability limit is $\sqrt{3}$ ). Scalar-excursion statistics are compared in fig. 18. The maximum global excursions are identical and the volume fraction of the $5 \%$ excursion shows relatively small differences in the second half of the run, which are more likely the result of somewhat different turbulent flow evolution rather than numerics. 
745

746

747

\section{B Viscous-term effects}

The simulations attain a high Reynolds number flow in which almost all dissipation is provided by the subgrid-scale model. Figure 19 confirms that the impact of resolved-scale diffusion is negligible, since excursion statistics are identical for a pair of runs with and without the viscous term. The very small effect of resolved-scale diffusion is discernible in the first third of the run where the excursions and the volume fraction are somewhat smaller when the viscous term is active. Figure 19 provides confidence in the correct implementation of the viscous term in the present LES. 


\section{References}

Arakawa, A. and V. R. Lamb, 1977: Computational design of the basic dynamical processes of the UCLA general circulation model. Methods of Computational Physics, Academic Press, Vol. 17, $173-265$.

Beare, R. J., et al., 2006: An intercomparison of large-eddy simulations of the stable boundary layer. Boundary-Layer Meteorol., 118, 247-272.

Brown, A. R., M. K. MacVean, and P. J. Mason, 2000: The effects of numerical dissipation in large eddy simulations. J. Atmos. Sci., 57 (19), 3337-3348.

Chow, F. K. and P. Moin, 2003: A further study of numerical errors in large-eddy simulations. $J$. Comput. Phys., 184 (2), 366-380.

Chung, D. and G. Matheou, 2012: Direct numerical simulation of stationary homogeneous stratified sheared turbulence. J. Fluid Mech., 696, 434-467.

Chung, D. and G. Matheou, 2014: Large-eddy simulation of stratified turbulence. Part I: A vortexbased subgrid-scale model. J. Atmos. Sci., 71, 1863-1879.

Cook, A. W., W. Cabot, and P. L. Miller, 2004: The mixing transition in Rayleigh-Taylor instability. J. Fluid Mech., 511, 333-362.

Dimotakis, P. E., 2000: The mixing transition in turbulent flows. J. Fluid Mech., 409, 69-98.

Dimotakis, P. E., 2005: Turbulent mixing. Annu. Rev. Fluid Mech., 37, 329-356.

Dimotakis, P. E. and P. L. Miller, 1990: Some consequences of the boundedness of scalar fluctuations. Phys. Fluids, 2 (11), 1919-1920.

Fedioun, I., N. Lardjane, and I. Gökalp, 2001: Revisiting numerical errors in direct and large eddy simulations of turbulence: physical and spectral spaces analysis. J. Comput. Phys., 174 (2), $816-851$. 
Geurts, B. J., 2009: Analysis of errors occurring in large eddy simulation. Phil. Trans. R. Soc. A, 367 (1899), 2873-2883.

Ghosal, S., 1996: An analysis of numerical errors in large-eddy simulations of turbulence. J. Comput. Phys., 125, 187-206.

Glaze, D. J., 2006: Large eddy simulation of a turbulent jet diffusion flame using the filtered mass density function model. Ph.D. thesis, Purdue University.

Harlow, F. H. and J. E. Welch, 1965: Numerical calculation of time-dependent viscous incompressible flow of fluid with free surface. Phys. Fluids, 8 (12), 2182-2189.

Hill, D. J. and D. I. Pullin, 2004: Hybrid tuned center-difference-WENO method for large eddy simulations in the presence of strong shocks. J. Comput. Phys., 194 (2), 435-450.

Horiuti, K., 1987: Comparison of conservative and rotational forms in large eddy simulation of turbulent channel flow. J. Comput. Phys., 71 (2), 343-370.

Inoue, M., G. Matheou, and J. Teixeira, 2014: LES of a spatially developing atmospheric boundary layer: Application of a fringe method for the stratocumulus to shallow cumulus cloud transition. Mon. Weather Rev., 142 (9), 3418-3424.

Jiang, G.-S. and C.-W. Shu, 1996: Efficient implementation of weighted ENO schemes. J. Comput. Phys., 126, 202-228.

Kartha, A., P. K. Subbareddy, G. V. Candler, and P. Dimotakis, 2015: LES of reacting mixing layers: Species concentration boundedness and inflow conditions. AIAA paper, AIAA-2015-320\%.

Kravchenko, A. G. and P. Moin, 1997: On the effect of numerical errors in large eddy simulations of turbulent flows. J. Comput. Phys., 131 (2), 310-322.

Leonard, B. P., 1979: A stable and accurate convective modelling procedure based on quadratic upstream interpolation. Comput. Methods in Appl. Mech. Eng., 19 (1), 59-98.

Lesieur, M. and O. Metais, 1996: New trends in large-eddy simulations of turbulence. Annu. Rev. Fluid Mech., 28, 45-82. 
LeVeque, R. J., 2002: Finite volume methods for hyperbolic problems. Cambridge university press.

Lilly, D. K., 1967: The representation of small-scale turbulence in numerical simulation experiments. Proc. IBM Sci. Computing Symp. Environmental Sci, 195-210.

Liu, X.-D., S. Osher, and T. Chan, 1994: Weighted essentially non-oscillatory schemes. J. Comput. Phys., 115 (1), 200-212.

Lomax, H., T. H. Pulliam, and D. W. Zingg, 2003: Fundamentals of Computational Fluid Dynamics. Scientific Computation, Springer.

Lundgren, T. S., 1982: Strained spiral vortex model for turbulent fine structure. Phys. Fluids, 25, 2193-2203.

Matheou, G., A. M. Bonanos, C. Pantano, and P. E. Dimotakis, 2010: Large-eddy simulation of mixing in a recirculating shear flow. J. Fluid Mech., 646, 375-414.

Matheou, G. and K. W. Bowman, 2016: A recycling method for the large-eddy simulation of plumes in the atmospheric boundary layer. Environmental Fluid Mechanics, 16, 69-85.

Matheou, G. and D. Chung, 2014: Large-eddy simulation of stratified turbulence. Part II: Application of the stretched-vortex model to the atmospheric boundary layer. J. Atmos. Sci., 71 (12), 4439-4460.

Matheou, G., D. Chung, L. Nuijens, B. Stevens, and J. Teixeira, 2011: On the fidelity of large-eddy simulation of shallow precipitating cumulus convection. Mon. Weather Rev., 139, 2918-2939.

Mattner, T. W., 2011: Large-eddy simulations of turbulent mixing layers using the stretched-vortex model. J. Fluid Mech., 671, 507-534.

Meinke, M., W. Schröder, E. Krause, and T. Rister, 2002: A comparison of second-and sixth-order methods for large-eddy simulations. Computers $\&$ Fluids, 31 (4), 695-718.

Metais, O. and M. Lesieur, 1992: Spectral large-eddy simulation of isotropic and stably stratified turbulence. J. Fluid Mech., 239, 157-194. 
Misra, A. and D. I. Pullin, 1997: A vortex-based subgrid stress model for large-eddy simulation. Phys. Fluids, 9, 2443-2454.

Mittal, R. and P. Moin, 1997: Suitability of upwind-biased finite difference schemes for large-eddy simulation of turbulent flows. AIAA journal, 35 (8), 1415-1417.

Moeng, C.-H. and J. C. Wyngaard, 1988: Spectral analysis of large-eddy simulations of the convective boundary layer. J. Atmos. Sci., 45 (23), 3573-3587.

Monaghan, J. J., 1988: An introduction to SPH. Comput. Phys. Commun., 48 (1), 89-96.

Morinishi, Y., T. S. Lund, O. V. Vasilyev, and P. Moin, 1998: Fully conservative higher order finite difference schemes for incompressible flow. J. Comput. Phys., 143 (1), 90-124.

Nadukandi, P., E. Oñate, and J. Garcia, 2010: A high-resolution petrov-galerkin method for the 1d convection-diffusion-reaction problem. Comput. Methods in Appl. Mech. Eng., 199 (9), 525-546.

Orszag, S. A., 1971: On the elimination of aliasing in finite-difference schemes by filtering highwavenumber components. J. Atmos. Sci., 28 (6), 1074-1074.

Pantano, C., D. I. Pullin, P. Dimotakis, and G. Matheou, 2008: LES approach for high Reynolds number wall-bounded flows with application to turbulent channel flow. J. Comput. Phys., 227, 9271-9291.

Park, N., J. Y. Yoo, and H. Choi, 2004: Discretization errors in large eddy simulation: on the suitability of centered and upwind-biased compact difference schemes. J. Comput. Phys., 198 (2), $580-616$.

Pope, S. B., 2004: Ten questions concerning the large-eddy simulation of turbulent flows. New Journal of Physics, 6, 35.

Pullin, D. I., 2000: A vortex-based model for the subgrid flux of a passive scalar. Phys. Fluids, 12, $2311-2316$.

Pullin, D. I. and P. G. Saffman, 1994: Reynolds stresses and one-dimensional spectra for a vortex model of homogeneous anisotropic turbulence. Phys. Fluids, 6, 1787-1796. 
Purnell, D., 1976: Solution of the advective equation by upstream interpolation with a cubic spline. Mon. Weather Rev., 104 (1), 42-48.

Rogallo, R. S., 1981: Numerical experiments in homogeneous turbulence. NASA Tech. Memo., 81315 .

Sagaut, P., 2006: Large Eddy Simulation for Incompressible Flows. 3d ed., Springer.

Schumann, U., 1985: Algorithms for direct numerical simulation of shear-periodic turbulence. Lecture Notes in Physics, Springer, Berlin, Vol. 218, 492-496.

Sengupta, T. K., G. Ganerwal, and A. Dipankar, 2004: High accuracy compact schemes and Gibbs' phenomenon. Journal of Scientific Computing, 21 (3), 253-268.

Shchepetkin, A. F. and J. C. McWilliams, 1998: Quasi-monotone advection schemes based on explicit locally adaptive dissipation. Mon. Weather Rev., 126 (6), 1541-1580.

Smagorinsky, J., 1963: General circulation experiments with the primitive equations. I. The basic experiment. Mon. Weather Rev., 91, 99-164.

Smolarkiewicz, P. K. and J. Szmelter, 2005: Mpdata: An edge-based unstructured-grid formulation. J. Comput. Phys., 206 (2), 624-649.

Smolarkiewicz, P. K. and J. Szmelter, 2009: Iterated upwind schemes for gas dynamics. J. Comput. Phys., 228 (1), 33-54.

Spalart, P. R., R. D. Moser, and M. M. Rogers, 1991: Spectral methods for the Navier-Stokes equations with one infinite and two periodic directions. J. Comput. Phys., 96 (2), 297-324.

Staniforth, A. and J. Côté, 1991: Semi-lagrangian integration schemes for atmospheric models-a review. Mon. Weather Rev., 119 (9), 2206-2223.

Subbareddy, P. K., G. V. Candler, and P. Ferrero, 2014: Scalar conservation in large eddy simulations of reacting flows. AIAA paper, AIAA-2014-3203.

Taylor, G. I., 1935: Statistical theory of turbulence. Proc. Roy. Soc. London, 151A, 421-478. 
Toro, E. F., 1999: Riemann Solvers and Numerical Methods for Fluid Dynamics. Springer.

Van Leer, B., 1977: Towards the ultimate conservative difference scheme III. Upstream-centered finite-difference schemes for ideal compressible flow. J. Comput. Phys., 23 (3), 263-275.

Verma, S., Y. Xuan, and G. Blanquart, 2014: An improved bounded semi-lagrangian scheme for the turbulent transport of passive scalars. J. Comput. Phys., 272, 1-22.

Voelkl, T., D. I. Pullin, and D. C. Chan, 2000: A physical-space version of the stretched-vortex subgrid-stress model for large-eddy simulation. Phys. Fluids, 12, 1810-1825.

Vreman, B., B. Geurts, and H. Kuerten, 1996: Comparision of numerical schemes in large-eddy simulation of the temporal mixing layer. Int. J. Numer. Methods Fluids, 22 (4), 297-311.

Williamson, D. L. and P. J. Rasch, 1989: Two-dimensional semi-lagrangian transport with shapepreserving interpolation. Mon. Weather Rev., 117 (1), 102-129.

Yoon, H. Y., S. Koshizuka, and Y. Oka, 1999: A particle-gridless hybrid method for incompressible flows. Int. J. Numer. Meth. Fluids, 30, 407-424.

Zhang, X. and C.-W. Shu, 2010: On maximum-principle-satisfying high order schemes for scalar conservation laws. J. Comput. Phys., 229 (9), 3091-3120. 


\section{List of Figures}

1 Modified wavenumber, $\kappa^{*}$, and numerical (modified) phase speed, $\alpha^{*}$, for the fully conservative second-, fourth- and sixth-order finite-difference schemes. An additional second-order scheme with a modified dispersion relation (labeled "mod 2nd") is also used to illustrate the effects different phase speed errors. . . . . . . . . . . . . 46

2 Numerical solutions to the one-dimensional linear convection equation with three convection schemes: second- and fourth-order fully conservative (left panel) and the modified second order. Dispersive oscillations develop near the solution gradients but the location of the oscillations (upwind or downwind of the gradient) depends on the numerical phase speed. Lines are as in fig. 1. . . . . . . . . . . . . . 47

3 Numerical solution (blue line) to the one-dimensional linear convection equation using a spectral approximation for the spatial derivative. Black line is the exact solution. Even though the spectral approximation is non-dispersive, small Gibbs under- and over-shoots are discernible because of the truncated approximation. Note the different character of the Gibbs oscillation with respect to dispersive oscillations in finite-difference approximations shown in fig. $2 . \ldots \ldots$. . . . . . . . . . 48

4 Evolution of the passive scalar field. $Z=1$ (red) fluid moves from left to right, $u=+1 \mathrm{~m} \mathrm{~s}^{-1}$, while $Z=0$ fluid (blue) moves from right to left, $u=-1 \mathrm{~m} \mathrm{~s}^{-1}$. . . 49

5 Evolution of the mixing layer width for the different convection schemes and SGS models for grid resolution $N=256$. For the stretched-vortex model $\Delta=\Delta x$, and for the Smagorinsky model $C_{s}=0.18$ is used. . . . . . . . . . . . . . 50

6 Resolved-scale passive scalar variance at $t=20 \mathrm{~s}$ for the different convection schemes and SGS models. The grid resolution is $N=256$. For the stretched-vortex model $\Delta=\Delta x$. Lines are as in fig. $5 . \ldots \ldots \ldots \ldots \ldots \ldots$

7 Normalized scalar dissipation rate at $t=20 \mathrm{~s}$ for the different convection schemes and SGS models. The grid resolution is $N=256$. Only the physical scalar dissipation rate is shown, i.e., without the contribution of the numerical dissipation. For the stretched-vortex model $\Delta=\Delta x$. Lines are as in fig. $5 . \ldots \ldots \ldots$. . . . . . . . 52 
8 Normalized scalar dissipation rate at $t=20 \mathrm{~s}$ for two resolutions and cutoff scales for runs with the stretched-vortex model. The fourth order convection scheme is used. 53

9 Time traces of global scalar excursion statistics with the fourth-order scheme, stretchedvortex model and $N=256$. The left panel shows the magnitude of under- and over-shoots (red), i.e., $-\min (\bar{Z})$ and $\max (\bar{Z})-1$. The right panel shows the volume fraction of $5 \%$ excursions. That is, the volume of fluid where $\bar{Z}>1.05$ (red) and

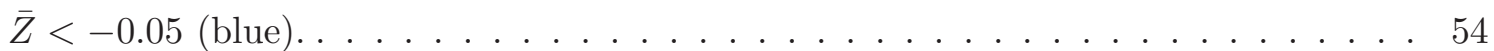

10 Global scalar excursion statistics for the different convection schemes (abscissa). The convection scheme symbols correspond to the order of accuracy of the fully conservative schemes, 'Q' corresponds to QUICK, 'S' to the pseudo-spectral approximation and ' $\mathrm{M}$ ' to the $\mathrm{MC}$ flux-limited monotone scheme. Blue symbols correspond to $N=256$, red to $N=512$, and a single magenta circle to $N=1024$. In the legend key, 'SMV $\Delta x$ ' corresponds to runs with the stretched-vortex SGS model with cutoff scale equal to the grid spacing, and 'SMV $2 \Delta x$ ' to double the grid spacing. Abbreviations SMG 0.18 and 0.23 correspond to runs with the Smagorinsky model with $C_{s}=0.18$ and 0.23 , respectively. . . . . . . . . . . . . 55

11 Probability density functions of the normalized scalar spatial derivatives for three grids, $N=256,512$, and 1024 . The sample size corresponds to 11 flow realizations in $t=10-20 \mathrm{~s}$, for runs with the fourth-order fully-conservative scheme and stretchedvortex model. . . . . . . . . . . . . . . . . . . . . 56

12 The left column panels show the magnitude of the scalar gradient (orange shading) and contours of scalar excursions at $\bar{Z}=-0.01$ (blue) and $\bar{Z}=1.01$ (red) on three planes. The right column panels show local excursions (orange contours) and the boundary of the mixed fluid (black contour) at the same planes as the corresponding left panel. All planes are at $t=20 \mathrm{~s}$ of the run with the fourth-order fully conservative scheme, stretched-vortex model and $N=256 \ldots \ldots \ldots$. . . . . . 57 
13 Magnitude of the scalar gradient (orange shading) and contour of scalar excursions at $\bar{Z}=-0.01$ (blue) and $\bar{Z}=1.01$ (red) on two planes from the run with the modified dispersion relation scheme, stretched-vortex model and $N=256$. A comparison with the corresponding panels of fig. 12 shows that some of the dispersive oscillations now occur upwind of the scalar gradient similar to right panel of fig. $2 . \quad \ldots \ldots$. . . . 58

14 Volume fraction of local excursions in the mixed fluid versus the total volume of excursions with respect to time. Two mixed-fluid thresholds are used, $0.1<\bar{Z}<0.99$ (red line) and $0.05<\bar{Z}<0.95 \ldots \ldots \ldots \ldots \ldots \ldots$

15 Two dimensional frequency distributions of local excursion-scalar gradient pairs for 11 flow realization in $t=10-20 \mathrm{~s}$ for a run with $N=256$, fourth order-convection and the stretched-vortex model. Darker shades correspond to higher frequencies. Because the frequency values span a large range of scales the contours are logarithmically spaced, i.e., $\log (h+1)$, where $h$ is the frequency of occurrence of a given pair. The left panel shows the co-located pairs, whereas in the right panel excursions are paired with the maximum scalar gradient magnitude in the surrounding $7 \times 7 \times 7$ grid cells. 60

16 Two dimensional frequency distributions of local excursion-scalar dissipation rate pairs for 11 flow realization in $t=10-20 \mathrm{~s}$ for a run with $N=256$, fourth orderconvection and the stretched-vortex model. Darker shades correspond to higher frequencies and contour levels are the same as in fig. 15. Because the frequency values span a large range of scales the contours are logarithmically spaced, i.e., $\log (h+1)$, where $h$ is the frequency of occurrence of a given pair. The left panel shows the co-located pairs, whereas in the right panel excursions are paired with the minimum scalar dissipation rate in the surrounding $7 \times 7 \times 7$ grid cells. . . . . . . 61

17 One dimensional scalar, $E_{Z Z}$ (top row), and scalar-gradient $k_{x}^{2} E_{Z Z}$, spectra along the streamwise direction on the mid-plane of the mixing layer for $N=256$ grids. Left panels corresponds to runs with the stretched-vortex model and right with the

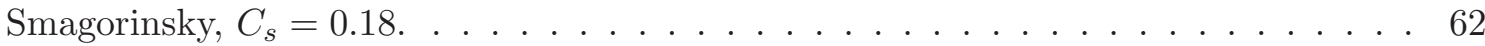


18 Effect of time step size on global excursion statistics. Time traces of scalar excursion statistics with the fourth-order scheme, stretched-vortex model, and $N=256$. Red lines correspond to simulations with $\mathrm{CFL}=1.4$ and blue lines to an identical LES setup but with a smaller time step, $\mathrm{CFL}=0.1$. The left panel shows the magnitude of global over-shoots. The right panel shows the volume fraction of $5 \%$ excursions. . 63

19 Impact of the viscous term on global excursion statistics. Time traces of scalar excursion statistics with the fourth-order scheme, stretched-vortex model, and $N=$ 256. Red lines correspond to simulations that include the viscous term and blue lines to an identical LES setup but without the viscous term. The left panel shows the magnitude of global over-shoots. The right panel shows the volume fraction of $5 \%$

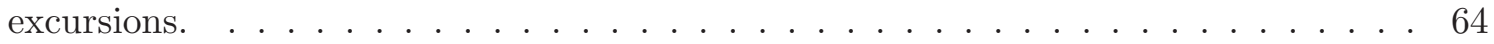




\section{List of Tables}

1 Summary of convection schemes. The columns correspond to the legend of the scalar convection scheme used in fig. 10, the scalar and momentum convection schemes, and the corresponding convection-scheme references. . . . . . . . . . . . . . 10

2 Scalar variance and kinetic energy conservation error with respect to time step size. The error decreases with the expected rate of $\Delta t^{3}$, the truncation error of the RungeKutta time integration. . . . . . . . . . . . . . . . 32 

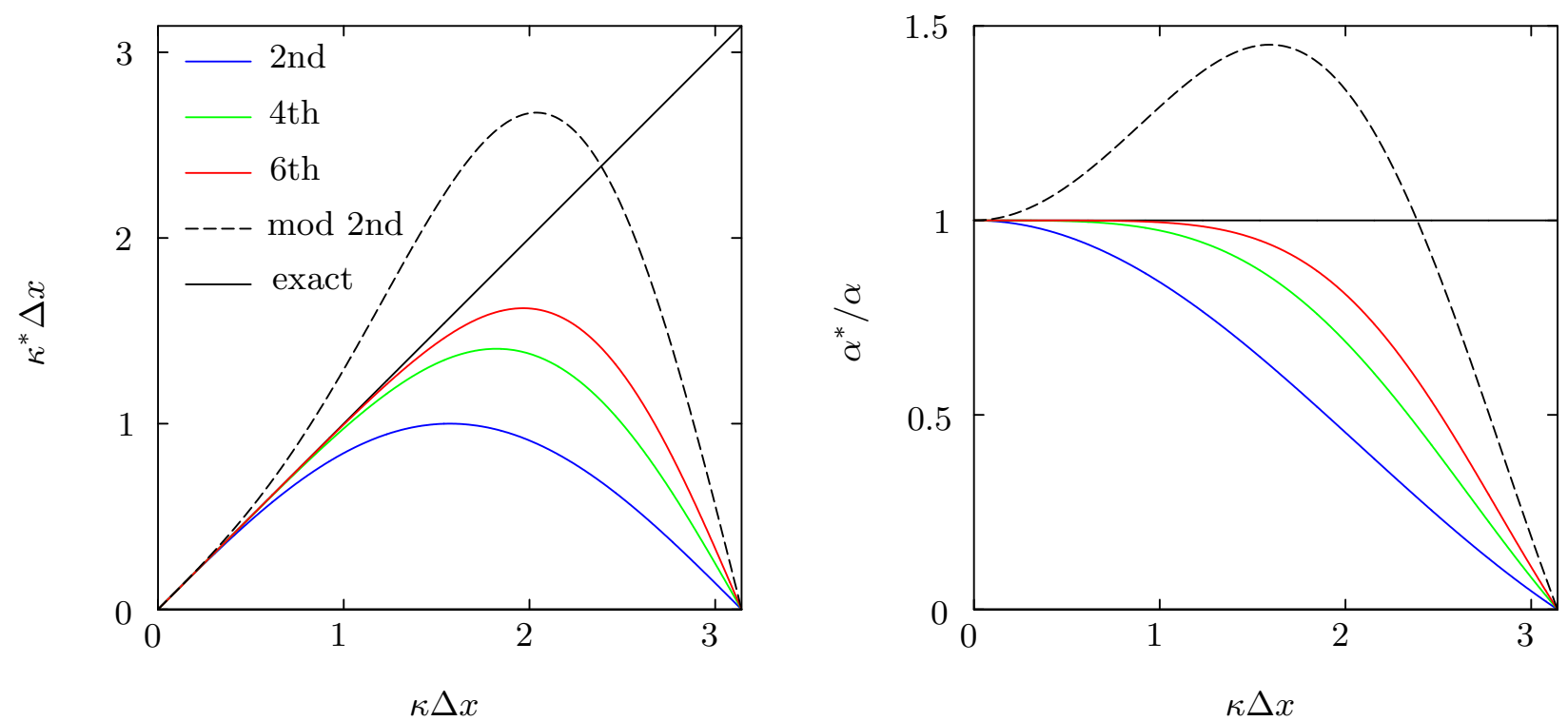

Figure 1: Modified wavenumber, $\kappa^{*}$, and numerical (modified) phase speed, $\alpha^{*}$, for the fully conservative second-, fourth- and sixth-order finite-difference schemes. An additional second-order scheme with a modified dispersion relation (labeled "mod 2nd") is also used to illustrate the effects different phase speed errors. 

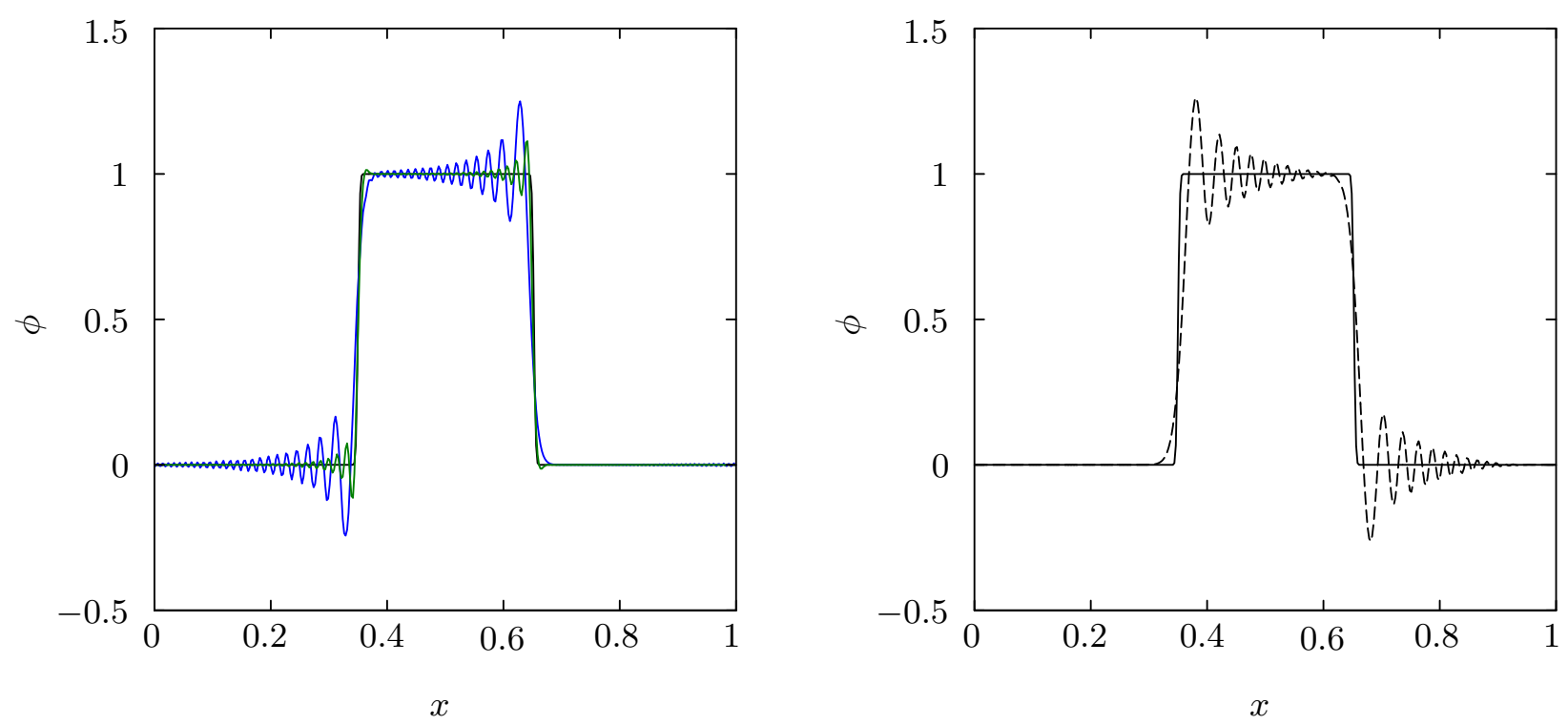

Figure 2: Numerical solutions to the one-dimensional linear convection equation with three convection schemes: second- and fourth-order fully conservative (left panel) and the modified second order. Dispersive oscillations develop near the solution gradients but the location of the oscillations (upwind or downwind of the gradient) depends on the numerical phase speed. Lines are as in fig. 1. 


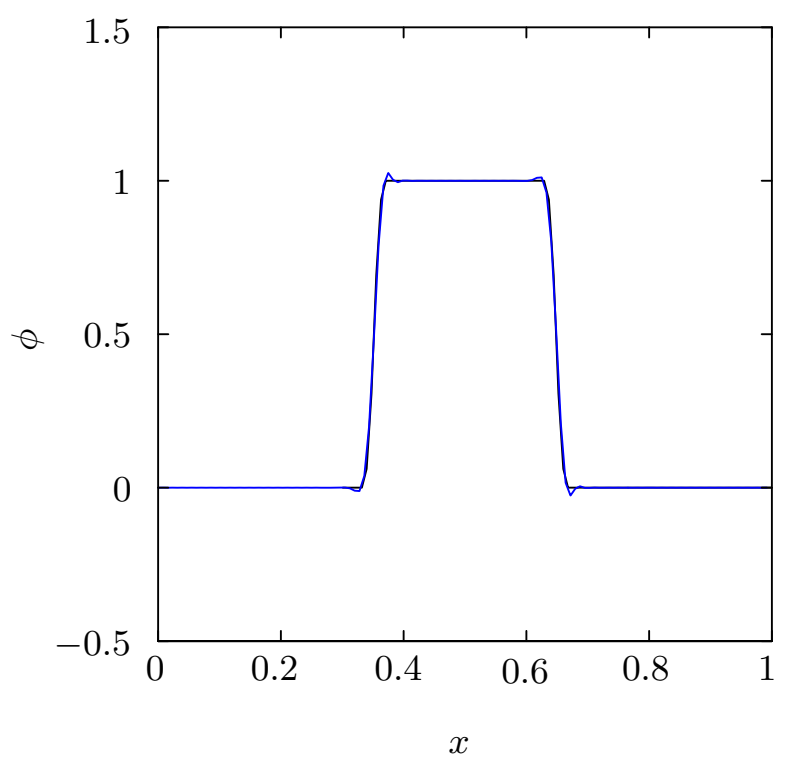

Figure 3: Numerical solution (blue line) to the one-dimensional linear convection equation using a spectral approximation for the spatial derivative. Black line is the exact solution. Even though the spectral approximation is non-dispersive, small Gibbs under- and over-shoots are discernible because of the truncated approximation. Note the different character of the Gibbs oscillation with respect to dispersive oscillations in finite-difference approximations shown in fig. 2. 

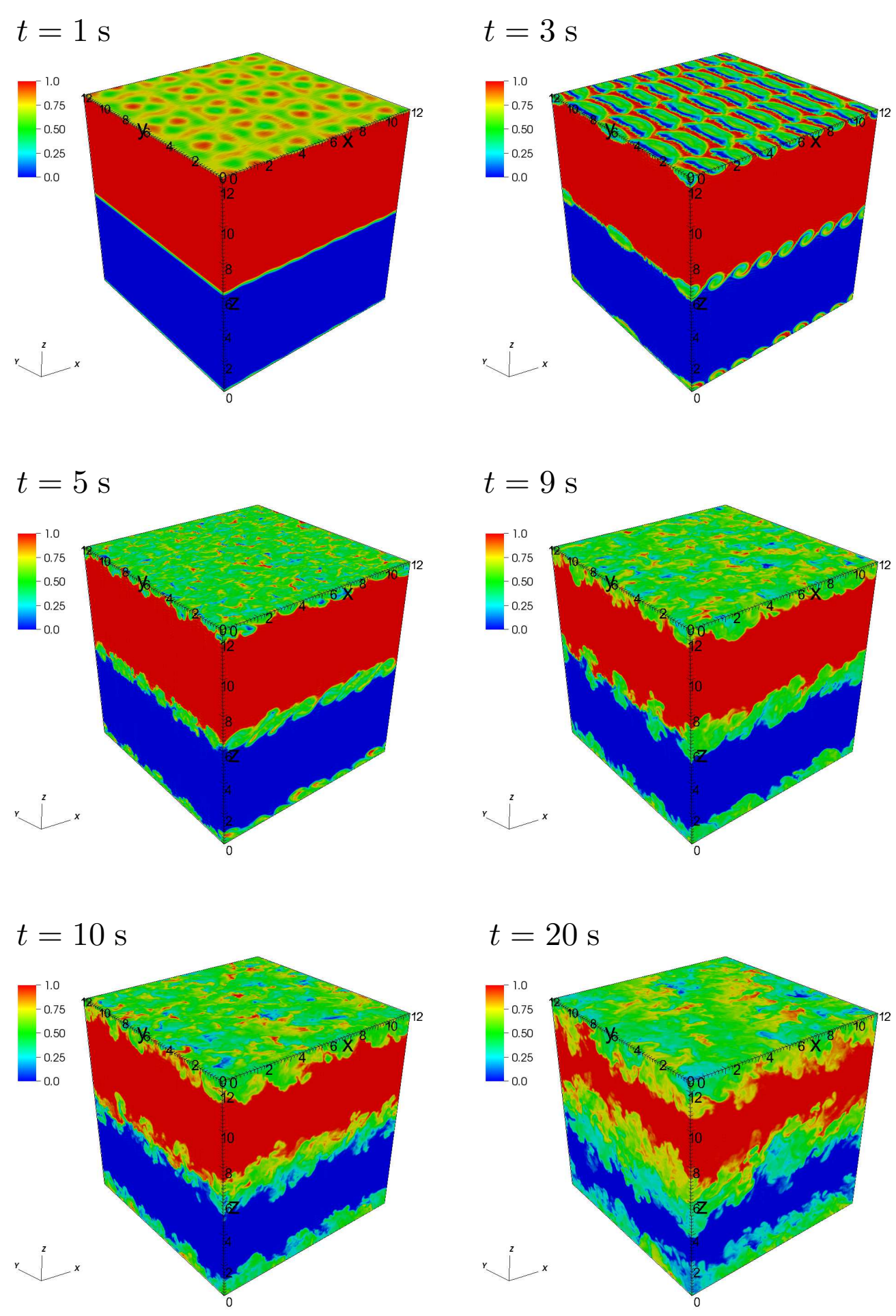

Figure 4: Evolution of the passive scalar field. $Z=1$ (red) fluid moves from left to right, $u=$ $+1 \mathrm{~m} \mathrm{~s}^{-1}$, while $Z=0$ fluid (blue) moves from right to left, $u=-1 \mathrm{~m} \mathrm{~s}^{-1}$. 

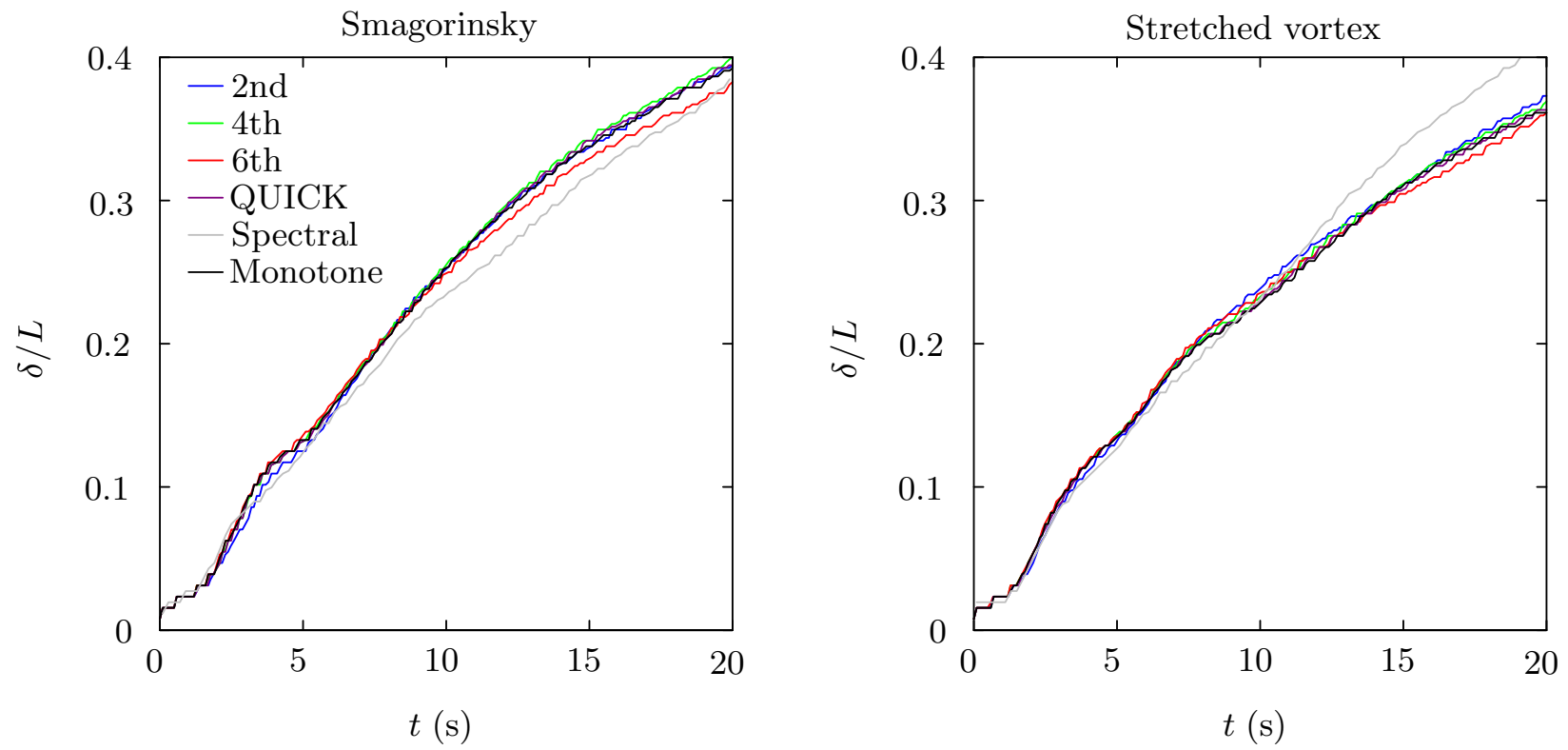

Figure 5: Evolution of the mixing layer width for the different convection schemes and SGS models for grid resolution $N=256$. For the stretched-vortex model $\Delta=\Delta x$, and for the Smagorinsky model $C_{s}=0.18$ is used. 

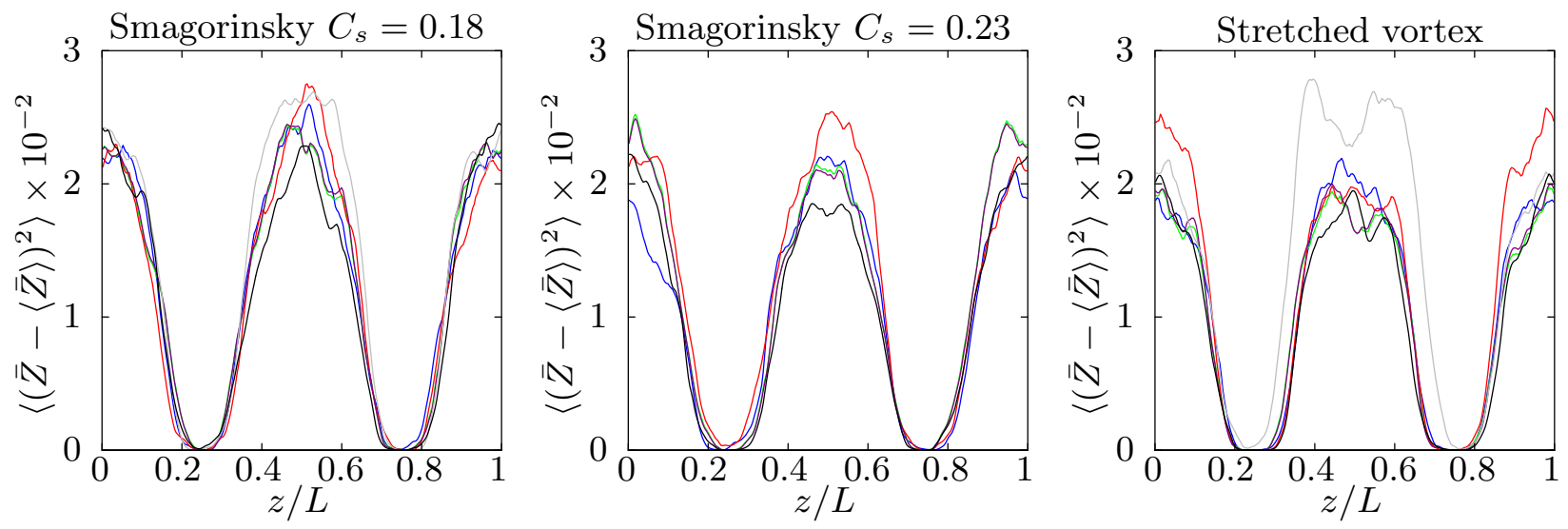

Figure 6: Resolved-scale passive scalar variance at $t=20 \mathrm{~s}$ for the different convection schemes and SGS models. The grid resolution is $N=256$. For the stretched-vortex model $\Delta=\Delta x$. Lines are as in fig. 5 . 

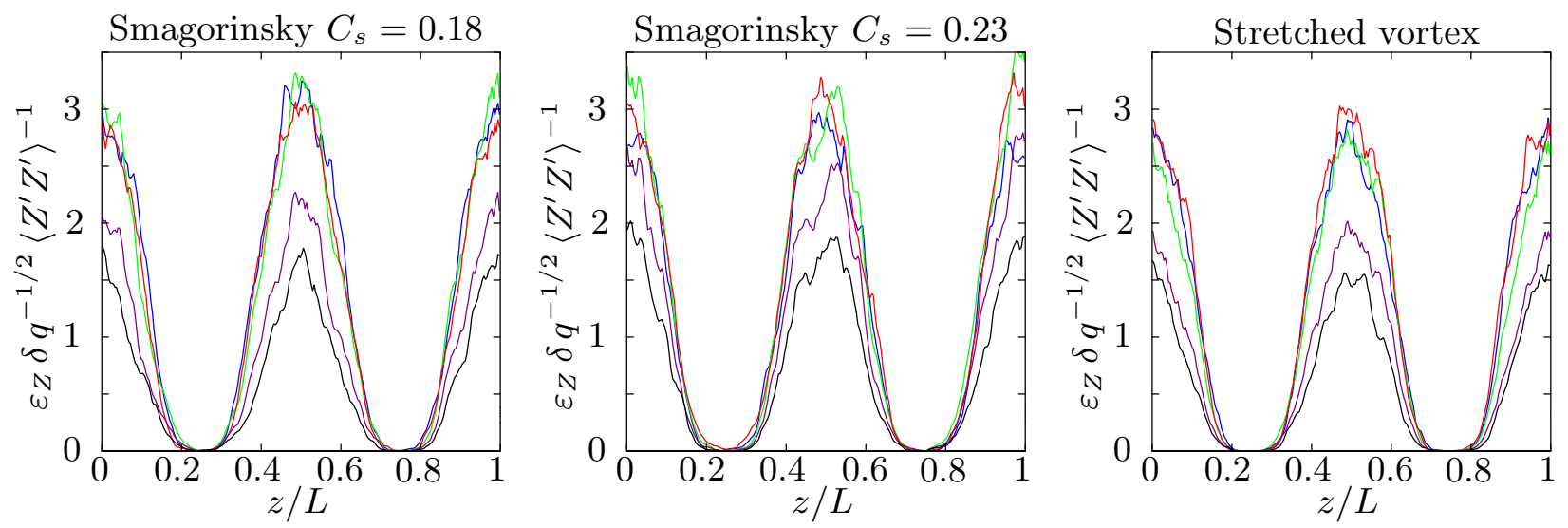

Figure 7: Normalized scalar dissipation rate at $t=20 \mathrm{~s}$ for the different convection schemes and SGS models. The grid resolution is $N=256$. Only the physical scalar dissipation rate is shown, i.e., without the contribution of the numerical dissipation. For the stretched-vortex model $\Delta=\Delta x$. Lines are as in fig. 5. 


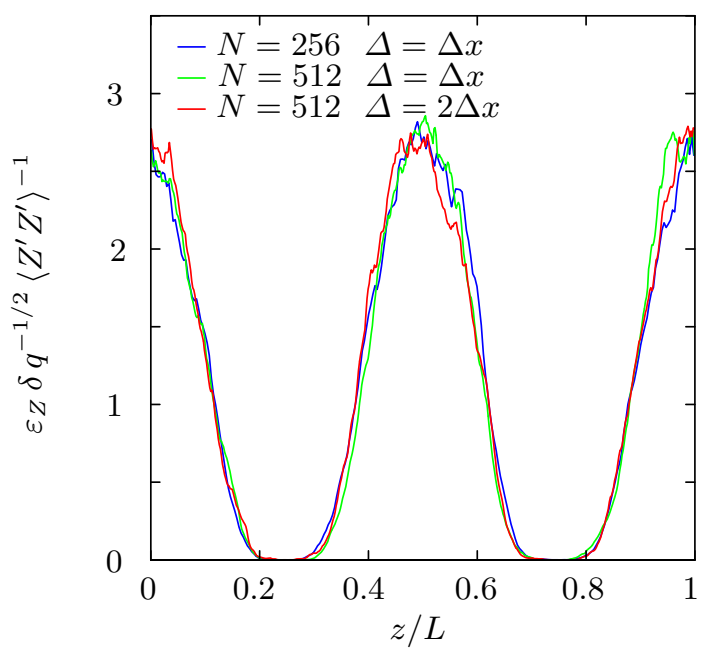

Figure 8: Normalized scalar dissipation rate at $t=20 \mathrm{~s}$ for two resolutions and cutoff scales for runs with the stretched-vortex model. The fourth order convection scheme is used. 

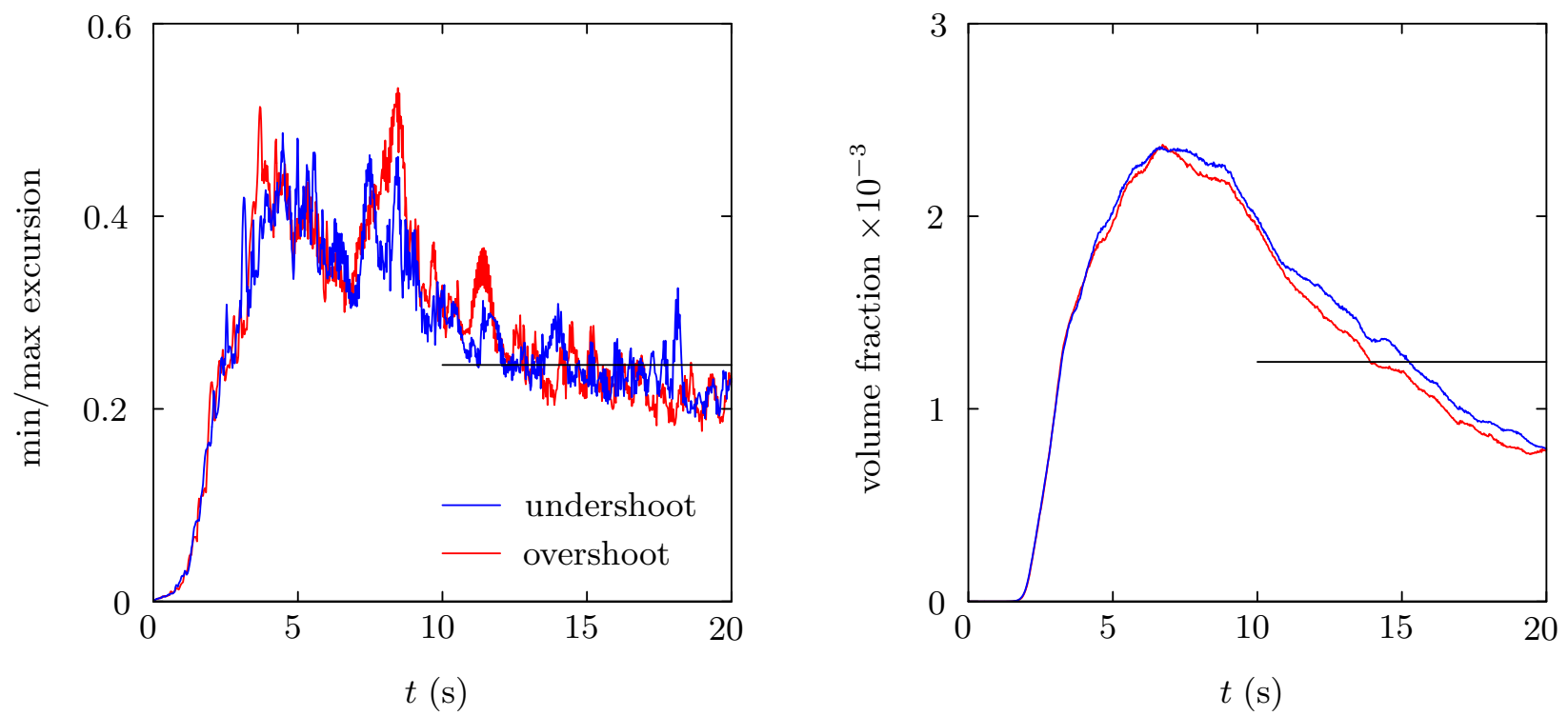

Figure 9: Time traces of global scalar excursion statistics with the fourth-order scheme, stretchedvortex model and $N=256$. The left panel shows the magnitude of under- and over-shoots (red), i.e., $-\min (\bar{Z})$ and $\max (\bar{Z})-1$. The right panel shows the volume fraction of $5 \%$ excursions. That is, the volume of fluid where $\bar{Z}>1.05$ (red) and $\bar{Z}<-0.05$ (blue). 

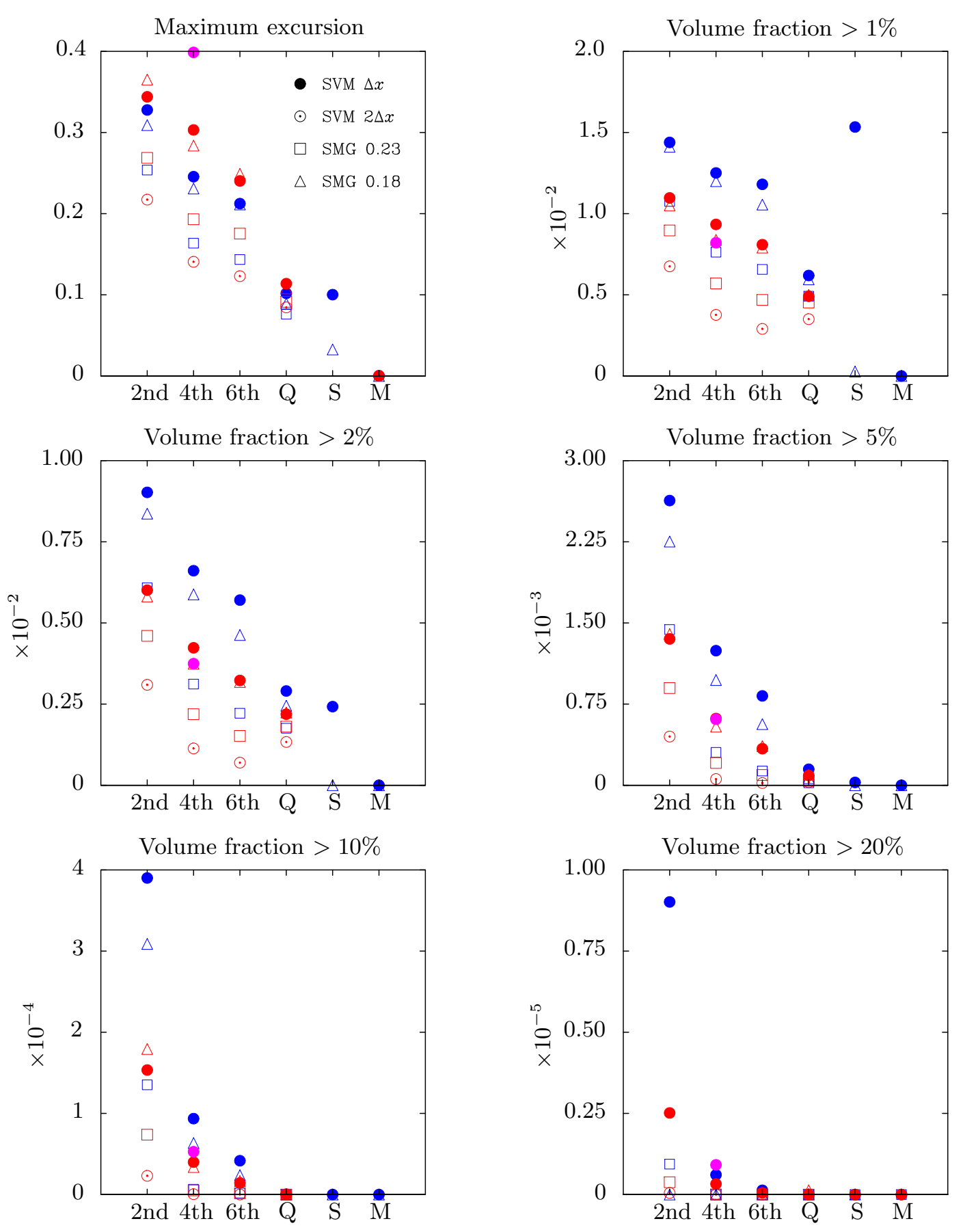

Figure 10: Global scalar excursion statistics for the different convection schemes (abscissa). The convection scheme symbols correspond to the order of accuracy of the fully conservative schemes, 'Q' corresponds to QUICK, 'S' to the pseudo-spectral approximation and ' $\mathrm{M}$ ' to the MC flux-limited monotone scheme. Blue symbols correspond to $N=256$, red to $N=512$, and a single magenta circle to $N=1024$. In the legend key, 'SMV $\Delta x$ ' corresponds to runs with the stretched-vortex SGS model with cutoff scale equal to the grid spacing, and 'SMV $2 \Delta x$ ' to double the grid spacing. Abbreviations SMG 0.18 and 0.23 correspond to runs with the Smagorinsky model with $C_{s}=0.18$ and 0.23 , respectively. 

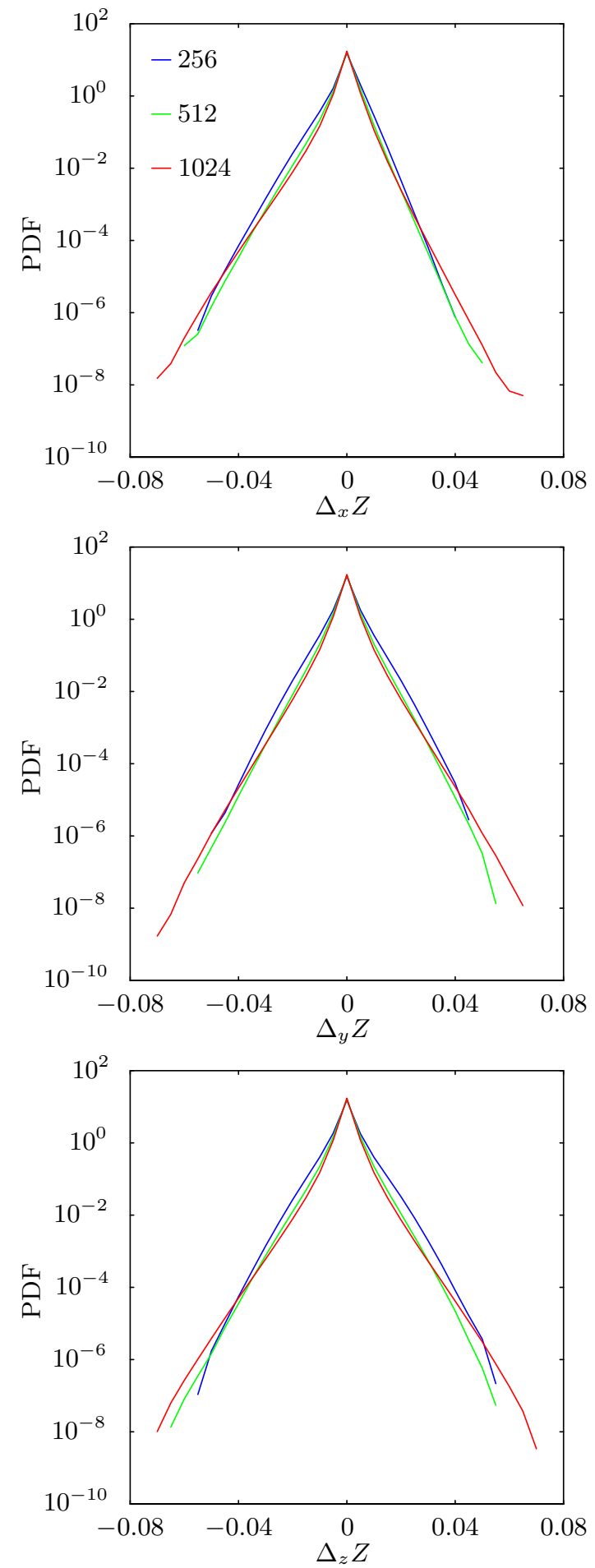

Figure 11: Probability density functions of the normalized scalar spatial derivatives for three grids, $N=256,512$, and 1024 . The sample size corresponds to 11 flow realizations in $t=10-20 \mathrm{~s}$, for runs with the fourth-order fully-conservative scheme and stretched-vortex model. 
$y=0 \mathrm{~m}$
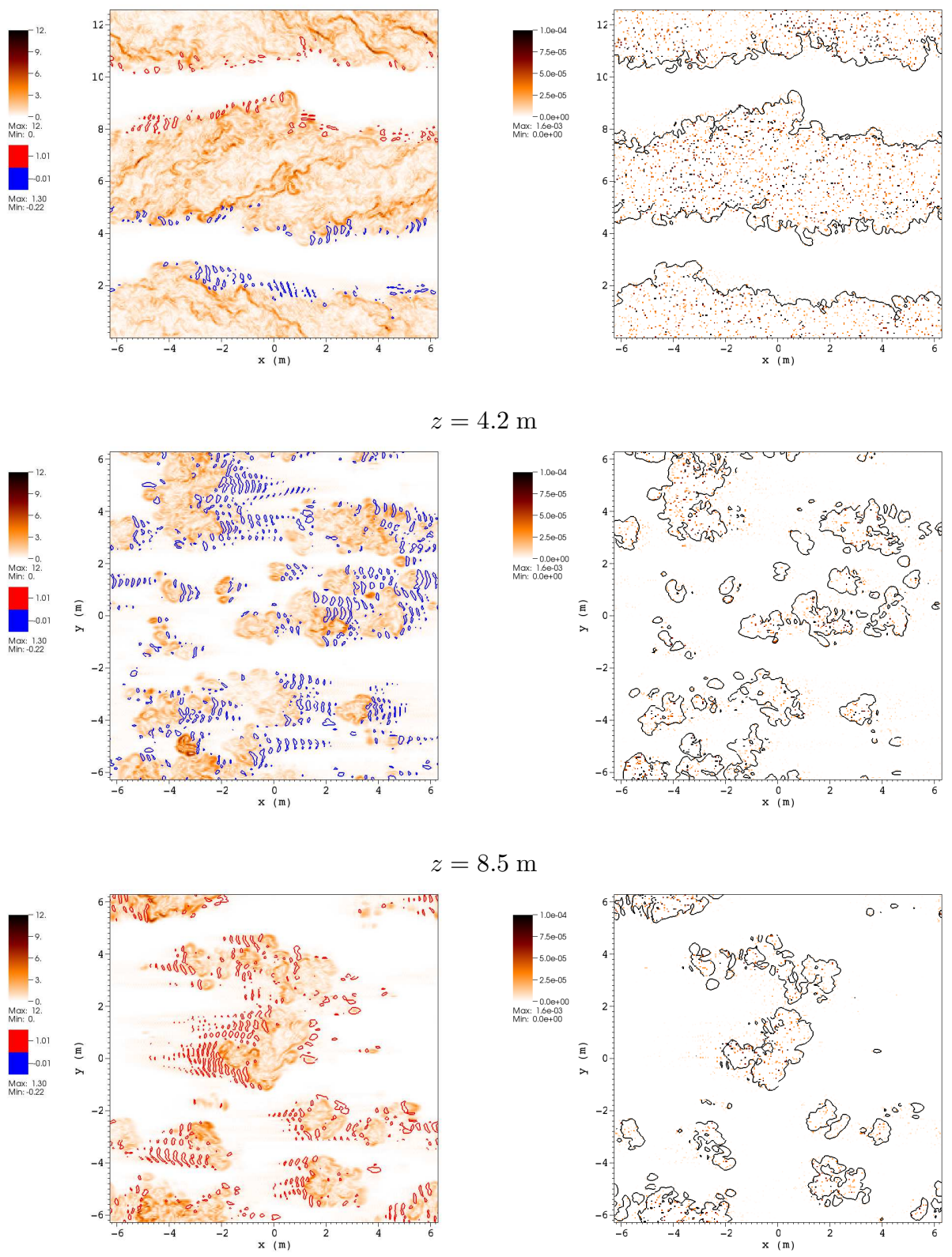

Figure 12: The left column panels show the magnitude of the scalar gradient (orange shading) and contours of scalar excursions at $\bar{Z}=-0.01$ (blue) and $\bar{Z}=1.01$ (red) on three planes. The right column panels show local excursions (orange contours) and the boundary of the mixed fluid (black contour) at the same planes as the corresponding left panel. All planes are at $t=20 \mathrm{~s}$ of the run with the fourth-order fully conservative scheme, stretched-vortex model and $N=256$. 

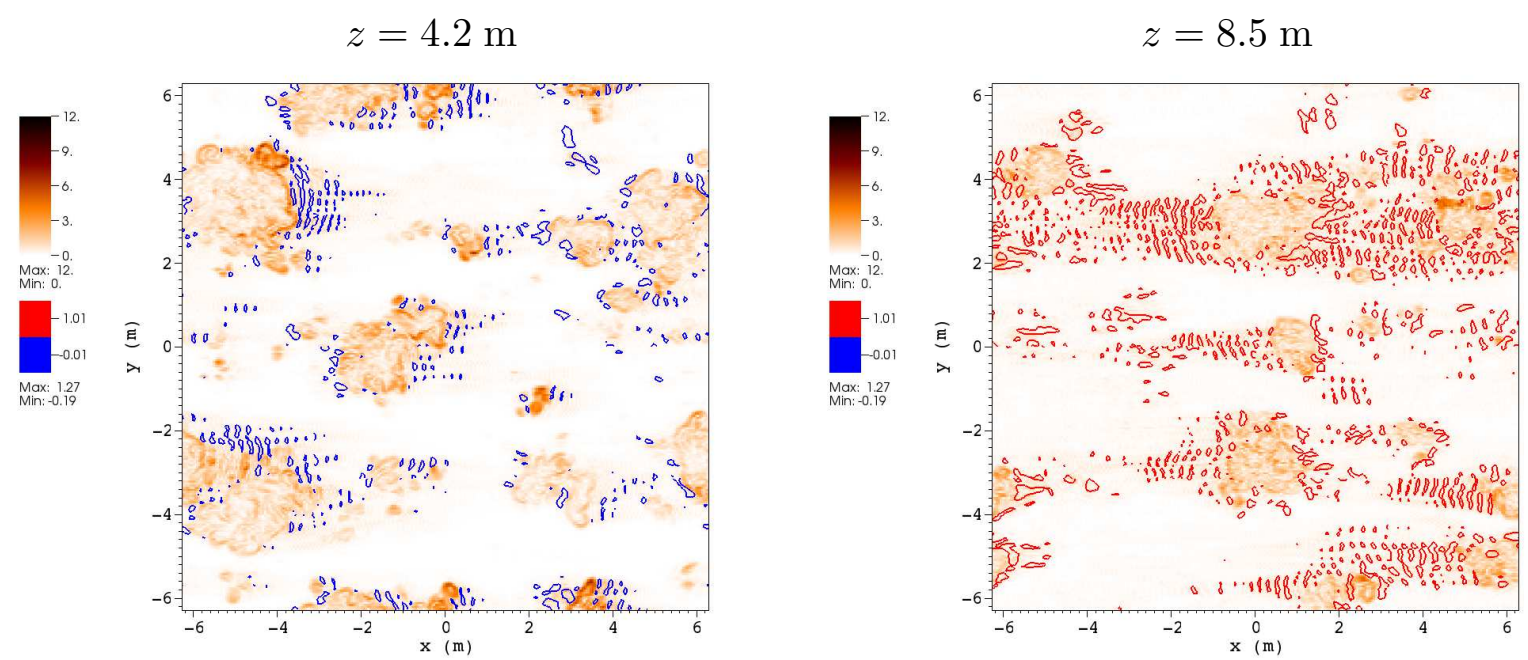

Figure 13: Magnitude of the scalar gradient (orange shading) and contour of scalar excursions at $\bar{Z}=-0.01$ (blue) and $\bar{Z}=1.01$ (red) on two planes from the run with the modified dispersion relation scheme, stretched-vortex model and $N=256$. A comparison with the corresponding panels of fig. 12 shows that some of the dispersive oscillations now occur upwind of the scalar gradient similar to right panel of fig. 2 . 


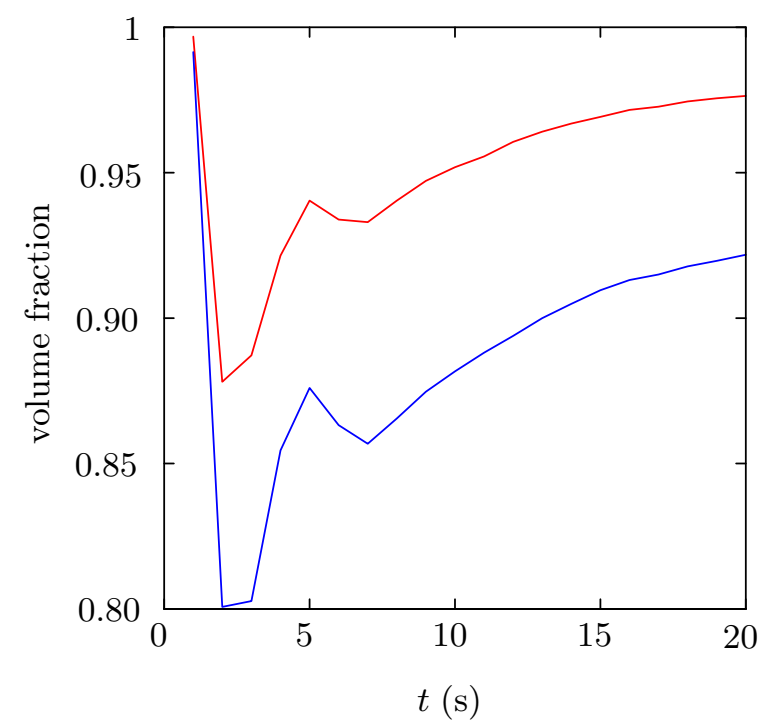

Figure 14: Volume fraction of local excursions in the mixed fluid versus the total volume of excursions with respect to time. Two mixed-fluid thresholds are used, $0.1<\bar{Z}<0.99$ (red line) and $0.05<\bar{Z}<0.95$. 

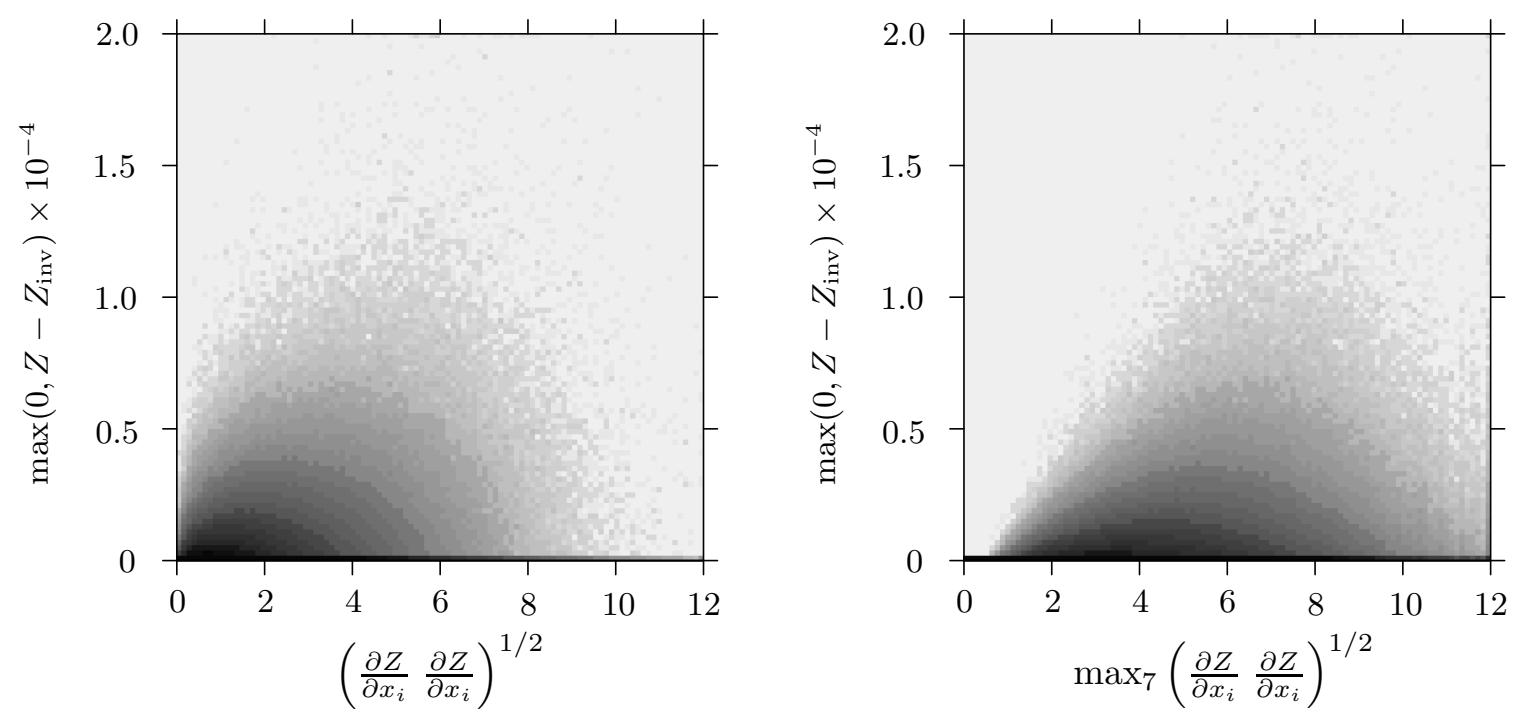

Figure 15: Two dimensional frequency distributions of local excursion-scalar gradient pairs for 11 flow realization in $t=10-20 \mathrm{~s}$ for a run with $N=256$, fourth order-convection and the stretchedvortex model. Darker shades correspond to higher frequencies. Because the frequency values span a large range of scales the contours are logarithmically spaced, i.e., $\log (h+1)$, where $h$ is the frequency of occurrence of a given pair. The left panel shows the co-located pairs, whereas in the right panel excursions are paired with the maximum scalar gradient magnitude in the surrounding $7 \times 7 \times 7$ grid cells 

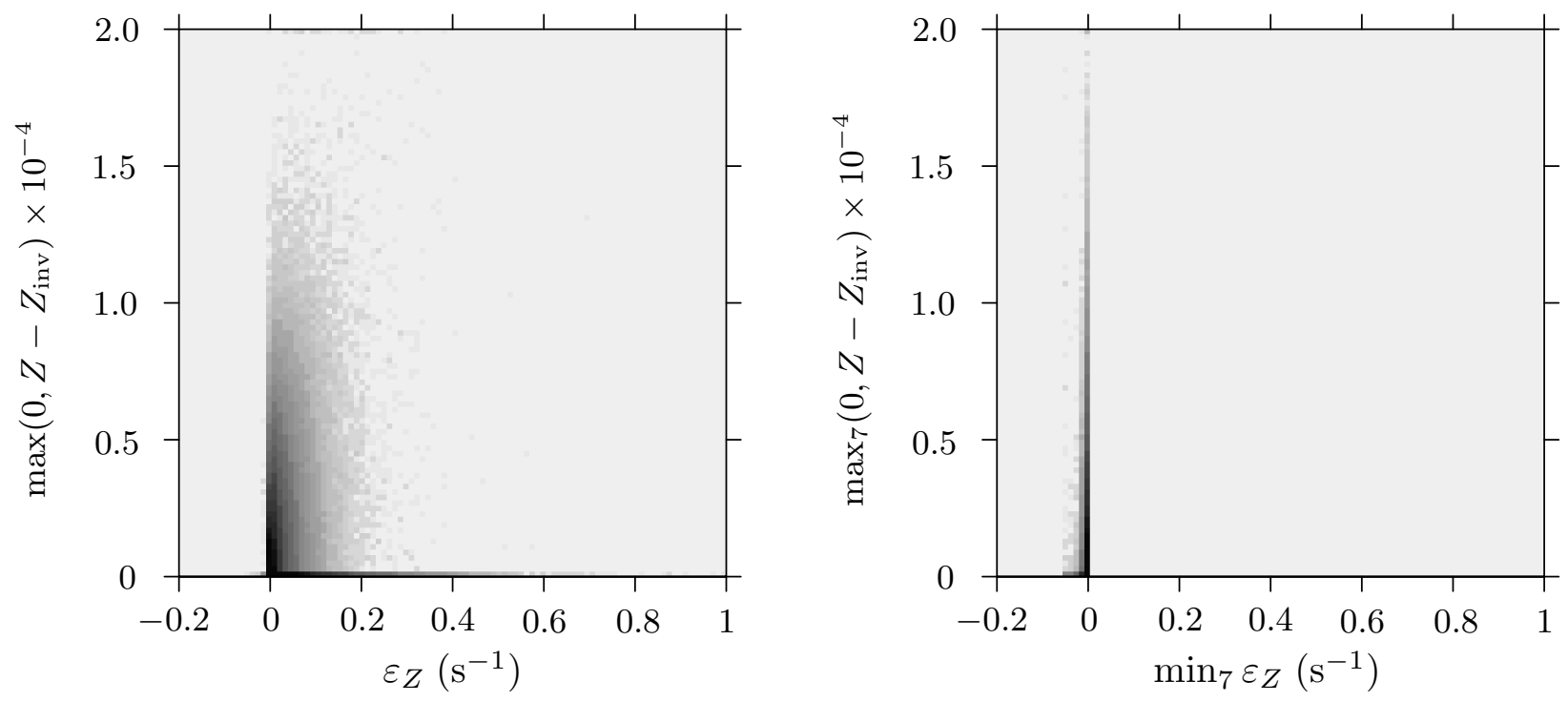

Figure 16: Two dimensional frequency distributions of local excursion-scalar dissipation rate pairs for 11 flow realization in $t=10-20 \mathrm{~s}$ for a run with $N=256$, fourth order-convection and the stretched-vortex model. Darker shades correspond to higher frequencies and contour levels are the same as in fig. 15. Because the frequency values span a large range of scales the contours are logarithmically spaced, i.e., $\log (h+1)$, where $h$ is the frequency of occurrence of a given pair. The left panel shows the co-located pairs, whereas in the right panel excursions are paired with the minimum scalar dissipation rate in the surrounding $7 \times 7 \times 7$ grid cells. 

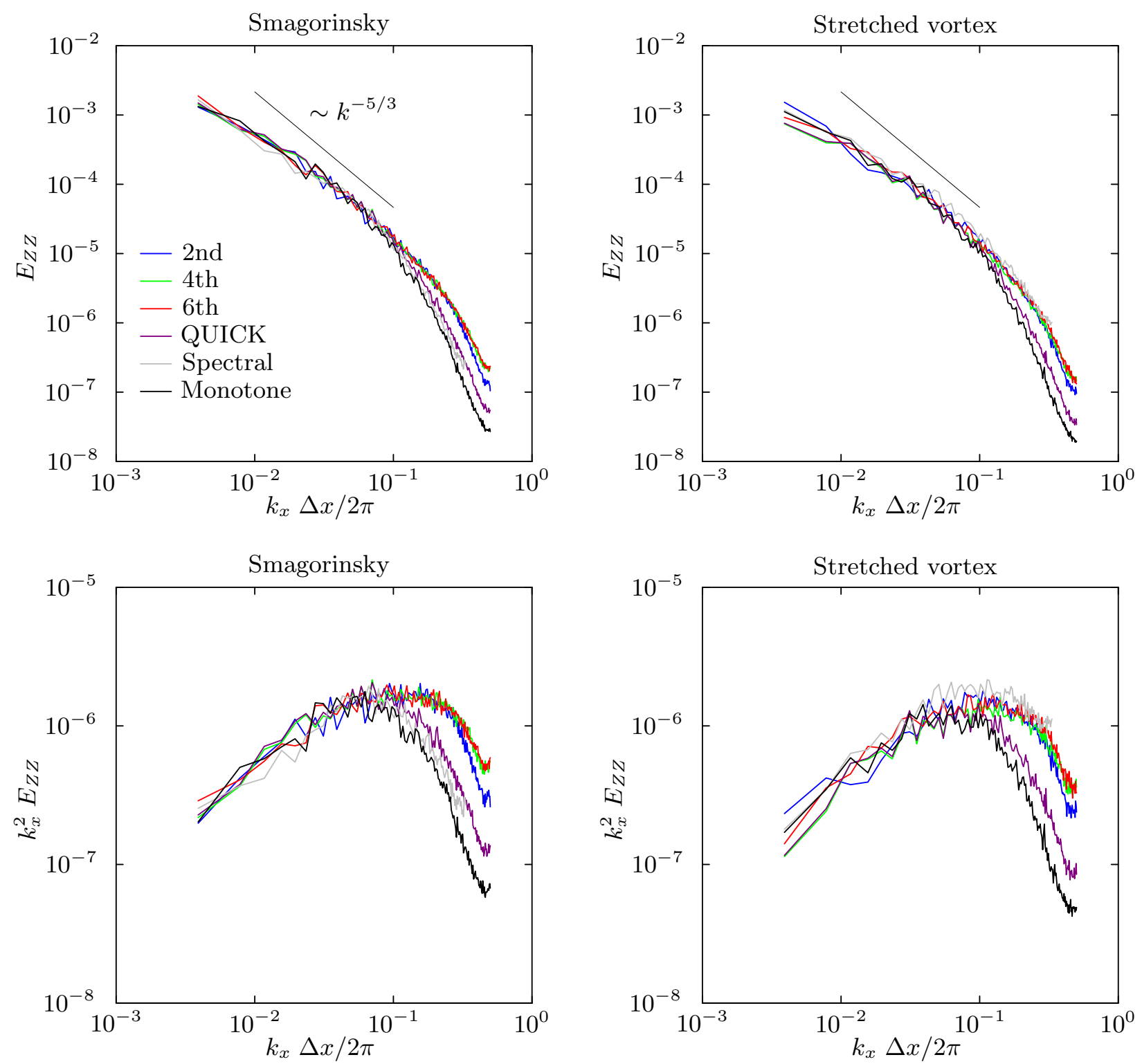

Figure 17: One dimensional scalar, $E_{Z Z}$ (top row), and scalar-gradient $k_{x}^{2} E_{Z Z}$, spectra along the streamwise direction on the mid-plane of the mixing layer for $N=256$ grids. Left panels corresponds to runs with the stretched-vortex model and right with the Smagorinsky, $C_{s}=0.18$. 

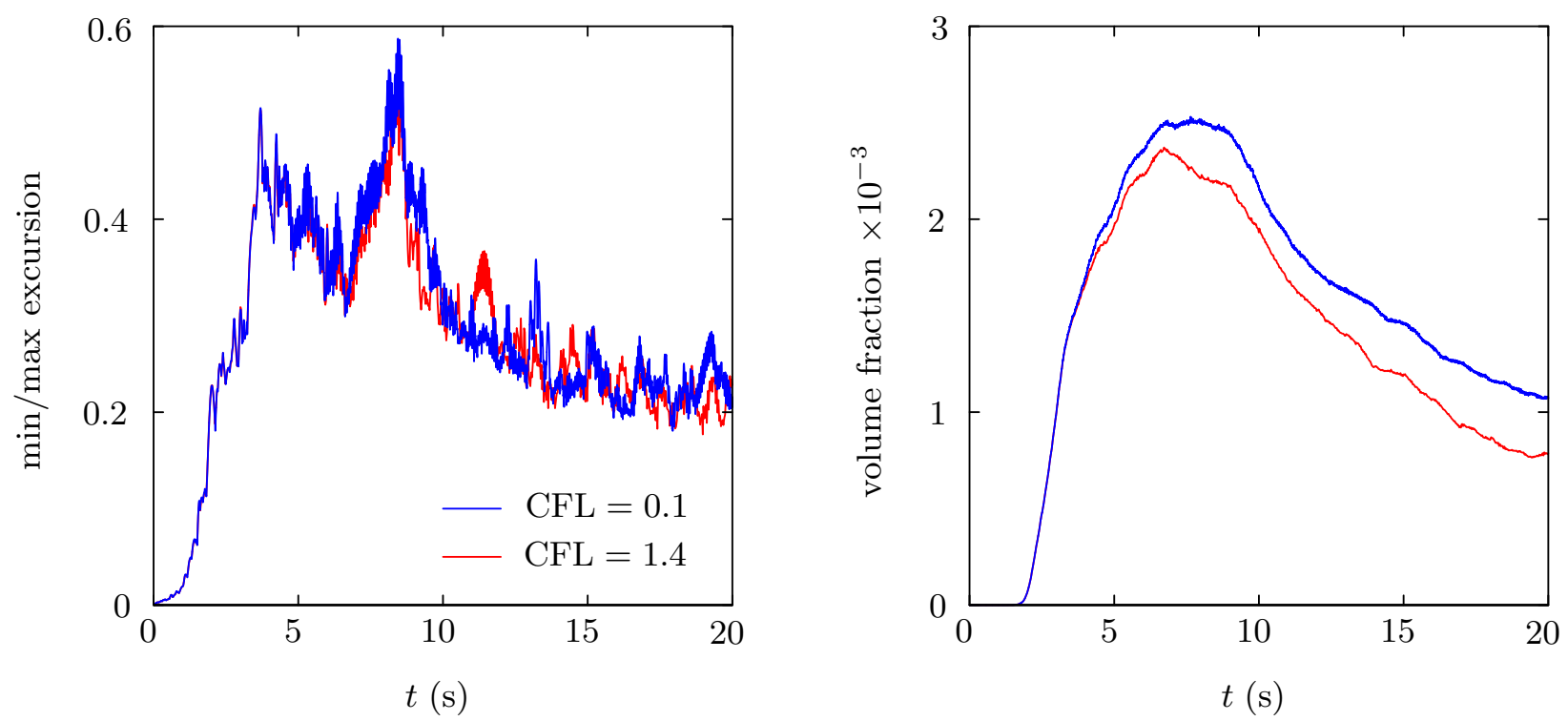

Figure 18: Effect of time step size on global excursion statistics. Time traces of scalar excursion statistics with the fourth-order scheme, stretched-vortex model, and $N=256$. Red lines correspond to simulations with $\mathrm{CFL}=1.4$ and blue lines to an identical LES setup but with a smaller time step, $\mathrm{CFL}=0.1$. The left panel shows the magnitude of global over-shoots. The right panel shows the volume fraction of $5 \%$ excursions. 

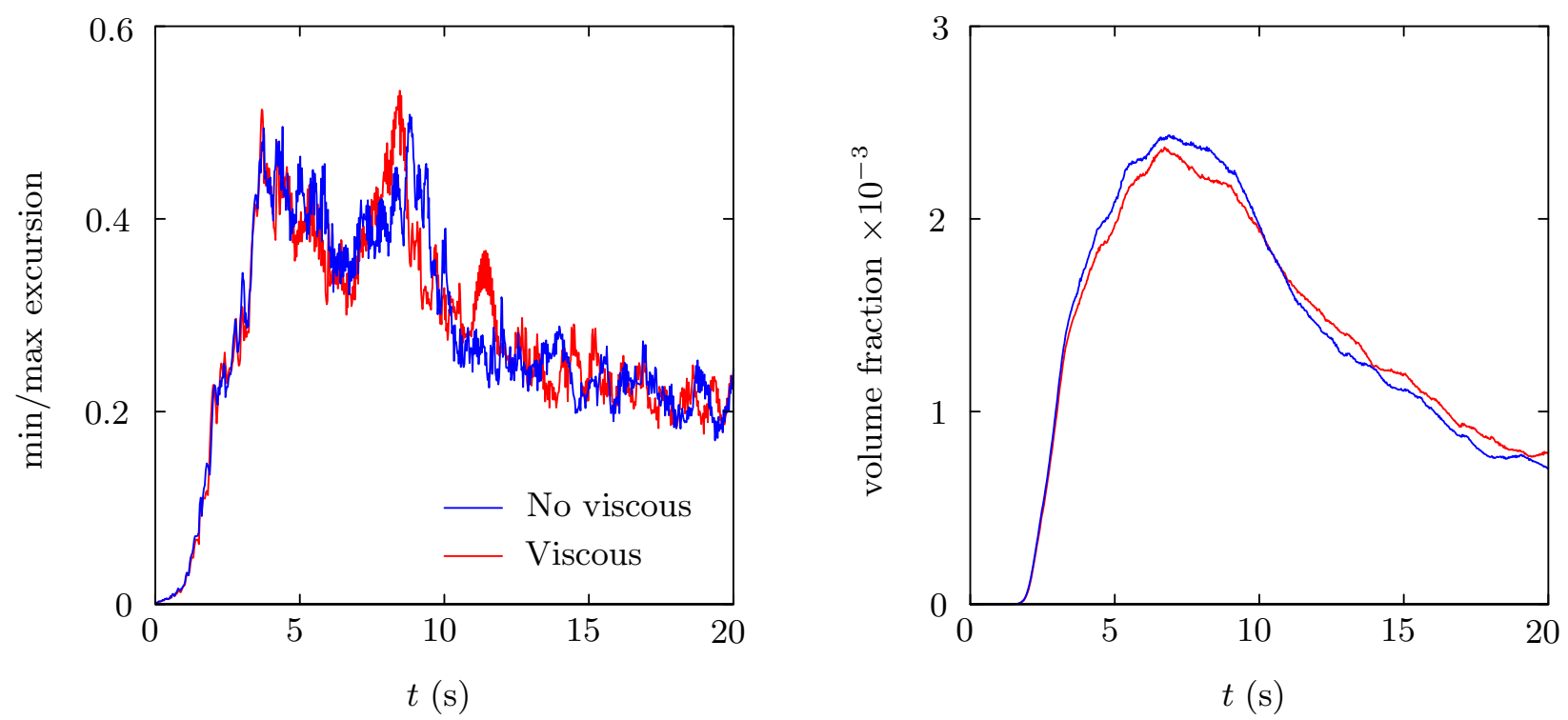

Figure 19: Impact of the viscous term on global excursion statistics. Time traces of scalar excursion statistics with the fourth-order scheme, stretched-vortex model, and $N=256$. Red lines correspond to simulations that include the viscous term and blue lines to an identical LES setup but without the viscous term. The left panel shows the magnitude of global over-shoots. The right panel shows the volume fraction of $5 \%$ excursions. 